\title{
Experiments, discrete and continuum simulations of the discharge of granular media from silos with a lateral orifice
}

\author{
Y. Zhou ${ }^{1,2}$, P.-Y. Lagrée ${ }^{3}$, S. Popinet ${ }^{3}$, P. Ruyer ${ }^{1}$ and \\ P. Aussillous ${ }^{2} \dagger$ \\ ${ }^{1}$ Institut de Radioprotection et de Sûreté Nucléaire (IRSN), PSN-RES, SEMIA, LIMAR, \\ Cadarache, St Paul-Lez-Durance, 13115, France \\ ${ }^{2}$ Aix-Marseille Univ, CNRS, IUSTI UMR 7343, 13013 Marseille, France \\ ${ }^{3}$ Sorbonne Universités, UPMC Univ Paris 06, CNRS UMR 7190, Institut Jean le Rond \\ $\partial^{\prime}$ Alembert, F-75005 Paris, France
}

(Received xx; revised xx; accepted xx)

We compare laboratory experiments, contact dynamics simulations, and continuum Navier-Stokes simulations with a $\mu(I)$ visco-plastic rheology, of the discharge of granular media from a silo with a lateral orifice. We consider a rectangular silo with an orifice of height $D$ which spans the silo width $W$, and we observe two regimes. For smallenough aperture aspect ratio $\mathcal{A}=D / W$, the Hagen-Beverloo relation is obtained. For thin-enough silos, $\mathcal{A} \gg \mathcal{A}_{c}$, we observe a second regime where the outlet velocity varies with $\sqrt{g W}$. This new regime is also obtained in the continuum simulations when the friction on side walls is taken into account in a thickness-averaged version of $\mu(I)+$ Navier-Stokes (in the spirit of Hele-Shaw flows). Moreover most of the internal details of the flow field observed experimentally are reproduced when considering this lateral friction. These two regimes are recovered experimentally for a cylindrical silo with a lateral rectangular orifice of height $D$ and $\operatorname{arc}$ length $W$. The dependency of the flow rate on the particle diameter is found to be reasonably described experimentally using two geometrical functions that depend respectively on the number of beads through the two aperture dimensions. This is consistent with $2 \mathrm{D}$ discrete simulation results: at the outlet, the volume fraction and the velocities depend on the particle diameter and this behaviour is correctly described by those geometrical functions. A similar dependency is observed in the $2 \mathrm{D}$ continuum simulations.

Key words: Dense granular flow, silo discharge, rheology

\section{Introduction}

Discharge of granular media out of a silo through an aperture is a classical problem with many practical and industrial applications. The main scaling relation, generally known as the Hagen-Beverloo relation, predicts that the mass flow rate scales as $(D-k d)^{5 / 2}$ where $D$ is the diameter of the aperture, $d$ the grain size and $k$ an empirical parameter. However detailed understanding of the physical processes leading to such a scaling is still lacking (Janda et al. 2012; Perge et al. 2012). Numerical simulations that explicitly solve

$\dagger$ Email address for correspondence: pascale.aussillous@univ-amu.fr 
granular media at the scale of the particle, like discrete element methods, can provide detailed and promising insight into the flow. On the other hand, rheological models able to describe the flow at the scale of its opening are still under development.

The Hagen-Beverloo scaling implies that, as long as the silo dimensions are sufficiently larger than the aperture scale, the geometry of the silo and its walls are irrelevant for the determination of the flow rate. However the conditions for which this statement holds are still unclear: what are the criteria that bound this behavior and what are the control parameters that drive the flow beyond the classical regime? The present study aims to study this departure for a specific case of industrial relevance. Let us consider a nonclassical geometrical configuration of the reservoir containing the granular medium: a vertical and elongated cylindrical tube of diameter of the order of centimeters with a lateral opening of similar size. This case is of particular interest to understand conditions under which solid fuel particles inside a typical Pressurised-Water Nuclear Reactor fuel rod, whose cladding would have failed under hypothetical accidental conditions, could disperse out of this rod. The fuel particles are generated from an initially cylindrical pellet (scale of centimeters) that can be fragmented due to the irradiation process (burnup of the fuel) or accidental conditions (large pressure and temperature variations inside the rod). The size distribution of the fragments can be wide, the smallest size being of the order of $10 \mu \mathrm{m}$. In this study the particles are composed of a population of spherical beads and are monodisperse in diameter. Therefore, the probability of jamming and arch formation throughout the silo is as low as possible due to the small contact area between neighboring particles. The discharge flow is therefore believed to overestimate that of a more realistic granular material of the same average size. This granular reservoir has two main peculiarities with regard to more classical hopper geometries. Firstly, for a given cross-section of the flow, the perimeter over which wall friction occurs is relatively large (by analogy, one could talk of a small hydraulic diameter) which raises the question of the possible impact of wall friction on the flow rate. Secondly, the aperture is vertical, an orientation that necessarily impacts the discharge for a gravity-driven flow. Moreover, the number of beads through the aperture can vary over a large range and is known to have a large effect on low values of flow rates.

The impact of the angle of the aperture surface (relative to horizontal) on the discharge flow rate of a silo (a so-called tilted hopper) has been already studied experimentally. For beads, Sheldon \& Durian (2010), or sand and sugar, Medina et al. (2014); Serrano et al. (2015) recovered a flow rate scaling as $D^{5 / 2}$, as long as clogging did not occur (which is only slightly affected by a vertical orientation of the aperture). According to the authors, the success of the Hagen-Beverloo scaling in this configuration indicates that one of the classical physical interpretations of the relation in terms of free-fall under an arch of aperture size is questionable. The shape and size of the aperture were varied, but the size of the aperture was always small compared to the silo width, which does not cover our range of interest. The wall thickness of the silo can alter the discharge flow rate of tilted hoppers as soon as it is sufficiently wide, according to Medina et al. (2014); Serrano et al. (2015). In these studies, the thickness was varied between zero and approximatively half the aperture size, the latter always being an order of magnitude larger than the typical grain size. In our case the typical cladding of a nuclear fuel rod is less than $1 \mathrm{~mm}$ thick and the experimental facility has been designed to avoid any impact of wall thickness on the discharge rate. In those studies, the number of grains in the aperture were varied solely by varying the aperture size, the grain size remaining constant. Sheldon \& Durian (2010) also underlined the possible influence of the hopper wall as being an interesting line for future research.

As long as the granular bed height inside the silo is larger than its width, the discharge 
flow rate does not depend on this height, a useful property used historically for time measurement with sandglasses. This has some similarity to the so-called Janssen effect that is observed for static packing of granular material confined by vertical walls. During the discharge, relative motion of the granular material with respect to these walls has to be considered and Bertho et al. (2003) have shown that the Janssen effect can be recovered in this configuration. However, Aguirre et al. (2010) have shown that the Janssen effect (i.e. the pressure level at the outlet) does not influence the granular discharge flow rate. The frictional interaction of flowing granular material with walls and its possible influence on the discharge flow rate for our geometry is therefore an open question.

For a small number of grains through the aperture, the flow rate depends on grain size (the larger the size, the lower the flow rate). The Hagen-Beverloo relation includes this effect as soon as $D / d<k$ (where $k$ is of order one). One of the classical interpretations of this $d$ dependency is the existence of an empty annulus that reduces the effective aperture area for the granular flow. But recent studies of the velocity and density profiles of the granular flow over horizontal apertures, e.g. Janda et al. (2012), indicate that the number of grains through the aperture is strongly correlated to the dilatancy of the flow over the entire flow cross section (and not only over its periphery). There is therefore interest in generalising this statement for other flow configurations where the Hagen-Beverloo relation holds, like the case of vertical aperture of interest in our study.

We present an investigation of the discharge flow rate of a granular material of spherical glass beads of variable size confined in a vertical elongated silo of variable shape and size, with a lateral aperture also of variable shape and size, while neglecting the effect of the wall thickness. The methods used are first introduced in section 2. The main scaling relations that could be deduced from dimensional analysis (see section 3.1) are recovered by the set of experiments performed (see section 3.2). It is then shown that the influence of the silo geometry on the flow rate can be simulated thanks to a continuum model for the granular flow with a rheology described by a $\mu(I)$ constitutive law and taking into account the wall friction (section 3.3 and 3.4). The observed influence of the beads size on the flow rate is analyzed in terms of dilatancy over the aperture cross-section: experimental results trends are supported by contact dynamics simulations of the flow through a vertical aperture (see section 3.5),

\section{Methods}

\subsection{Experiments}

Two geometries of silos have been considered, either rectangular or cylindrical as shown in figure 1(a,b). The typical height $H$ of the silos is larger than $500 \mathrm{~mm}$, that is always one order of magnitude larger than its lateral extent. The lateral extent of the rectangular silo and the diameter of the cylindrical silo are fixed to $60 \mathrm{~mm}$ and $40 \mathrm{~mm}$ respectively. The aperture on the lateral sidewall has a rectangular shape with a height $D$ and a horizontal length $W$. For rectangular silos, $W$ is also the width of the silo. For cylindrical silos, $W$ is the orifice arc length. The whole set of geometrical data considered in this study is given in table 1 . The back wall of the rectangular silo is made of a copper frame connected to ground to discharge static electricity. Front, bottom, and lateral walls of the rectangular silo, as well as cylindrical walls are made of a Plexiglas frame of millimeter width. The walls have been bevelled along the aperture with an angle $\delta$ as represented in figure 1 . A preliminary study has shown that the discharge flow rate was independent from this angle (and therefore that the friction along the wall thickness was negligible) as long as $\delta<60^{\circ}$. The value $\delta=30^{\circ}$ has been chosen. The bottom of the aperture is at a vertical 
(a)

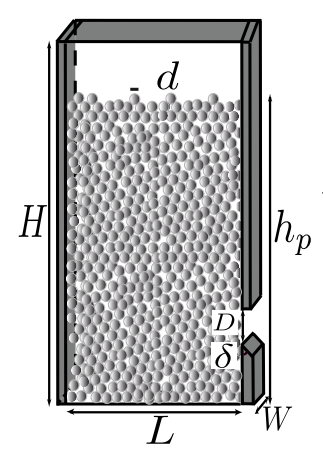

(b)

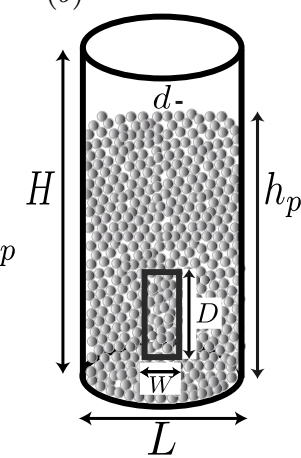

(c)

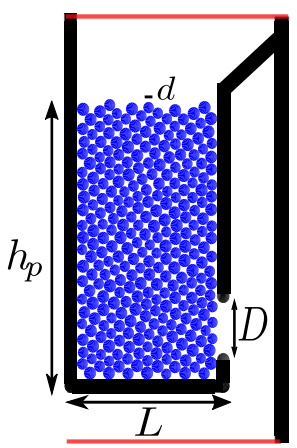

$(d)$

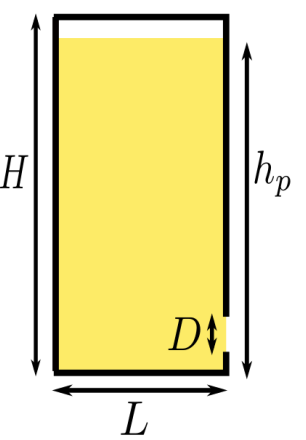

Figure 1: Schematic apparatus of the silo. (a) Experimental rectangular silo. (b) Experimental cylindrical silo. (c) 2D discrete simulation. (d) Continuum simulation. The red lines in figure $\mathrm{c}$ represent the horizontal boundaries of the computational domain.

Table 1: Performed runs, the dimension are defined in figure 1.

$D$

$$
\begin{gathered}
{[2.7,5.4,10,15} \\
20,25,30,35] \mathrm{mm} \\
{[5,10,20,25,30} \\
35,40,41.5] \mathrm{mm}
\end{gathered}
$$

Exp. Cyl. silo

Discrete 2D sim.

$W$

$$
\begin{array}{cc}
{\left[\begin{array}{c}
3.5,5,10,20 \\
30,40] \mathrm{mm}
\end{array}\right.} & {[124,190,375,538} \\
& \\
& \\
{[5,10.1,15.4,20.9} & {[124,190,375,538} \\
33.9,62.8] \mathrm{mm} & 762,1129,1347] \mu \mathrm{m}
\end{array}
$$

$$
[6,8,10,12
$$$$
[14,16: 18,20] \mathrm{d}
$$

Continuum $2 \mathrm{D} \operatorname{sim}$.

$$
\begin{aligned}
& {[0.4375,0.5,0.5625,0.625} \\
& 0.6562,0.6875,0.75] \mathrm{L}
\end{aligned}
$$

$[2,6] \mathrm{mm}$

$L / 30, L / 90$

$L / 30, L / 90$

Continuum $\quad[0.4375,0.5,0.5625,0.5938$

pseudo-3D sim. $0.625,0.6562,0.6875,0.75] \mathrm{L}$

$[0.16,0.2,0.25$
$0.5,1,2] \mathrm{L}$

distance larger than $20 \mathrm{~mm}$ from the bottom of the silo (corresponding to at least 15 layers of beads): lower values affect the discharge rate.

The non-cohesive spherical glass beads of density $\rho=2500 \mathrm{~kg} \mathrm{~m}^{-3}$ (Potter \& Ballotini Inc.) have been sifted between $0.9 d$ and $1.1 d$, with $d$ the mean diameter. The initial (before the opening of the aperture) volume fraction of particles in the silo $\phi_{b}$ has been estimated from the initial mass (of granular material before filling) and from the initial height $h_{p}$ within the silo. During the discharge, the grains flowing through the aperture are collected and their mass is measured with an electronic balance (Mettler Toldeo $6002 \mathrm{~S}$ ) with an accuracy of $0.1 \mathrm{~g}$ and a frequency of $20 \mathrm{~Hz}$. We observed a steady-state discharge regime for all the configurations explored. From the slope of the collected mass evolution during this regime, one deduces the instantaneous mass flow rate $Q_{i}$, and the mean flow rate, $Q$, as displayed in figure $2 \mathrm{a}$. The data are available as supplementary material in a text file. A Photron high-speed optical camera FASTCAM APX RS has been used during rectangular silo discharge with a spatial resolution of $256 \times 1024$ pixels $^{2}$, a frequency of acquisition of $250 \mathrm{~Hz}$ with a SIGMA zoom $24-700 \mathrm{~mm}$ f2.8. Using the 
$(a)$

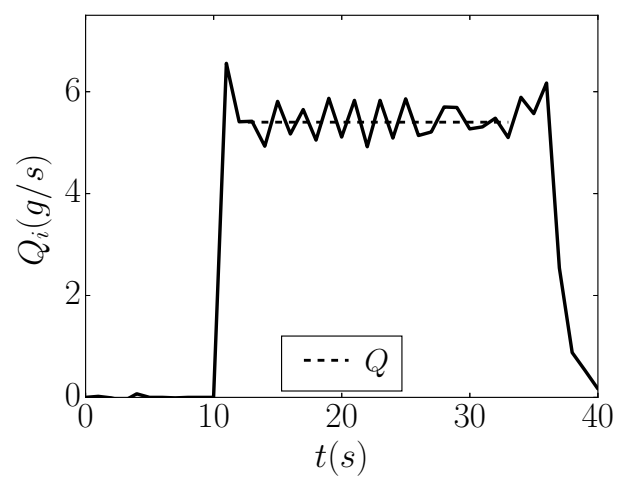

(b)

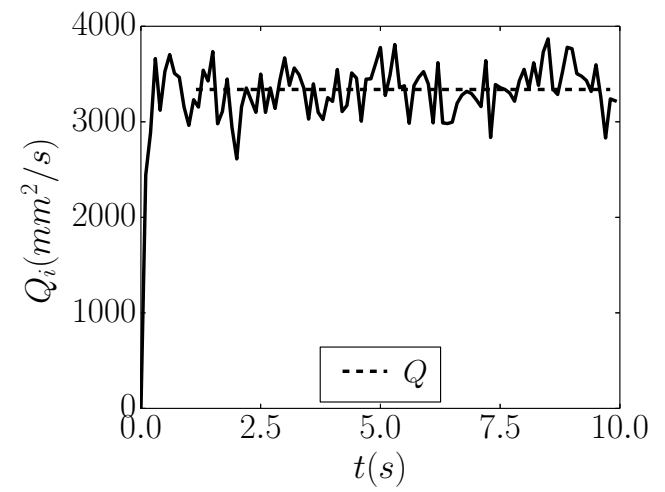

Figure 2: Temporal evolution of the instantaneous flow rate. (a) Experiment with rectangular silo, $d=190 \mu \mathrm{m}, D=10 \mathrm{~mm}$ and $W=3.5 \mathrm{~mm}$. (b) $2 \mathrm{D}$ discrete simulation with $d=2 \mathrm{~mm}$ and $D=16 \mathrm{~mm}$. The dashed lines represent the mean flow rate $Q$.

DPIVsoft software (Meunier \& Leweke 2003) we performed PIV of the granular flow and were able to get streamlines and $2 \mathrm{D}$ velocity fields at the front wall of the silo (as illustrated in figure 7). Using an interface tracking algorithm, the instantaneous profile of the upper layer of beads in the silo has also been recorded (as illustrated in figure 11).

\subsection{Contact Dynamics simulations}

Following the work of Zhou et al. (2015) we used the LMGC 90 software implementation of the contact dynamics method (Radjai \& Dubois 2011) to study the discharge of a silo with a lateral orifice. As discrete simulations in 3D are too demanding given our computational resources, we only performed 2D simulations, which still take hours or even days. The two-dimensional silo (figure 1c) consists of a rectangular reservoir, of width $L$, filled with a height $h_{p}$ of particles of mean size $d$. There is a dispersion $\delta d / d=0.2$ in the size of the particles to avoid crystallisation. The wall thickness is imposed to be equal to the diameter of the biggest particle in the silo, $d_{M}$, with a circular shape at the edge of the outlet. The outlet is located at the side, $3.5 d_{M}$ above the bottom, and has a length $D$ which was varied. The circular particles are treated as perfectly rigid and inelastic and contact dissipation is modelled in terms of a friction coefficient that we set to $\mu_{p}=0.4$ between particles, and $\mu_{w}=0.5$ between the particles and the wall. The number of particles, reported in table 2, was chosen for each simulation to ensure that the discharge flow rate is independent from the column height with $16 D<H<45 D$. To ensure that the lateral walls do not influence the flow significantly, we impose a width of the silo $L=3 D$. The granular column is prepared by the random deposition of particles in the closed silo. The initial volume fraction of particles in the silo $\phi_{b}$ has been measured in the central zone of the silo. Simulations are then run with a time step of $\delta t=5 \cdot 10^{-4} \mathrm{~s}$ and for a number of time steps $N_{t}$ reported in table 2. The computational domain is periodic in the vertical direction to keep a constant number of particles. The horizontal boundaries of the computational domain are set at a distance $10 d_{M}$ below, and $30 d_{M}$ to $70 d_{M}$ above the silo (see the red lines in figure 1c).

Figure $2 \mathrm{~b}$ shows a typical temporal evolution of the instantaneous flow rate. The flow rate is found to rapidly reach a stationary value $Q$. The output data at the aperture line are time averaged during this steady-state regime of discharge to deduce the vertical 
Table 2: Parameters used in discrete simulations for given particle sizes $(d=2 \mathrm{~mm}$ and $d=6 \mathrm{~mm}$ ), where $N_{p}$ is the number of particles and $N_{t}$ is the number of time steps.

\begin{tabular}{c||c|c||c|c|c|}
\hline \multicolumn{4}{|c||}{$\mid$} & $d=2 \mathrm{~mm}$ & \multicolumn{2}{c|}{$d=6 \mathrm{~mm}$} \\
\hline$D / d$ & $N_{p}$ & $N_{t}$ & $N_{p}$ & $N_{t} \mid$ \\
\hline 6 & 5000 & 30000 & 4000 & 30600 \\
8 & 7500 & 20000 & 5400 & 22000 \\
10 & 10000 & 20000 & 8400 & 20000 \\
12 & 11290 & 16000 & 12000 & 20000 \\
14 & 13500 & 15000 & 14000 & 16000 \\
16 & 15500 & 10000 & 16000 & 9200 \\
18 & 20000 & 5200 & 18000 & 8000 \\
20 & & & 20000 & 6000
\end{tabular}

profiles of velocity and particle volume fraction. Further details may be found in Zhou et al. (2015).

\subsection{Continuum numerical simulations}

\subsubsection{General equations}

We turn to the continuum simulation method in the framework of the $\mu(I)$-rheology, a non-Newtonian rheology for granular flows proposed by Midi (2004); Jop et al. (2006). The non-Newtonian incompressible Navier-Stokes system reads:

$$
\begin{gathered}
\boldsymbol{\nabla} \cdot \mathbf{u}=0, \\
\rho\left(\frac{\partial \mathbf{u}}{\partial t}+\mathbf{u} \cdot \nabla \mathbf{u}\right)=-\nabla p+\nabla \cdot(2 \eta \mathbf{D})+\rho \mathbf{g}+\mathbf{f}_{w},
\end{gathered}
$$

where $\mathbf{D}$ is the strain-rate tensor $\left(\boldsymbol{\nabla u}+\boldsymbol{\nabla} \mathbf{u}^{T}\right) / 2$ and $\mathbf{f}_{w}$ is a volumetric force (discussed in section 2.3.3). Following Jop et al. (2005), the $\mu(I)$ viscosity is an effective viscosity $\eta$ depending both on the shear-rate (the inertial number $I$ being proportional to the second invariant $D_{2}$ of the strain rate tensor $D_{2}=\sqrt{D_{i j} D_{i j}}$ ) and the local pressure. It reads:

$$
\eta=\frac{\mu(I)}{\sqrt{2} D_{2}} p, \text { with } I=\frac{d \sqrt{2} D_{2}}{\sqrt{p / \rho}}, \quad \text { and } \mu(I)=\mu_{s}+\frac{\Delta \mu}{I_{0} / I+1} .
$$

We take for the rheological constants $\mu_{s}=0.4, \Delta \mu=0.28$ and $I_{0}=0.4$, but we do not consider the variation of the volume fraction with $I$ given by Jop et al. (2006), as we suppose the flow incompressible. At the solid boundaries we impose a no-slip condition. Pressure is zero at the orifice. In the original model pressure is zero at the free surface. To simplify the implementation of this boundary condition for a moving free surface, we introduce a second passive fluid of small density and viscosity and impose the zero pressure condition along the top boundary of the domain.

The Navier-Stokes simulations are performed with the free software $\mathcal{B} a$ silisk (Popinet 2013-2016), which is the successor of Gerris (Popinet 2003, 2009) and uses a similar finitevolume projection method. Two phases are present, a surrounding gas and the granular fluid. The interface between these two phases is tracked with a Volume-Of-Fluid method. The viscosity and density of the surrounding gas are small, so that its influence on the 
(a)

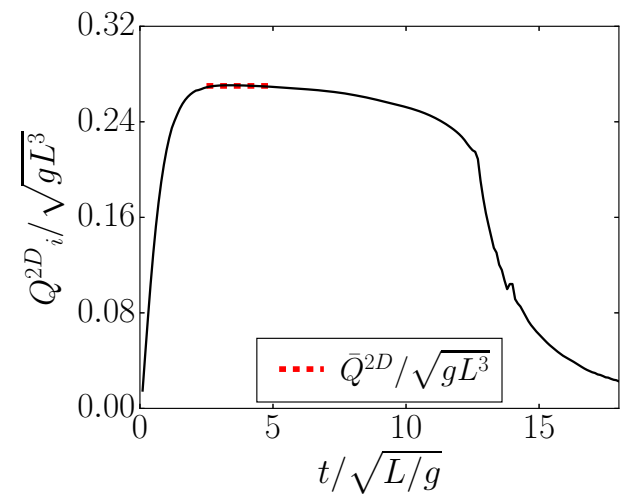

(b)

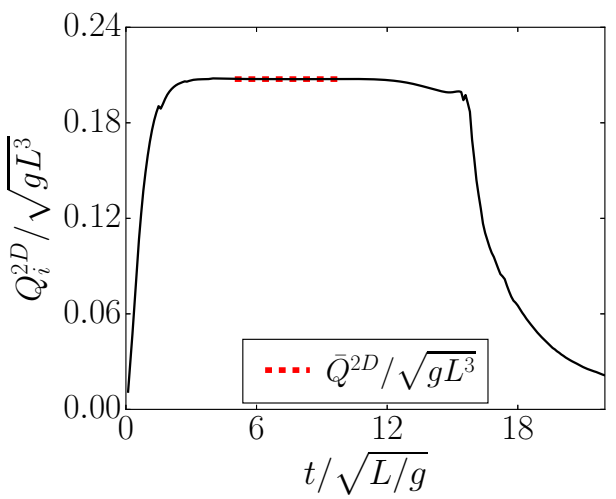

Figure 3: Continuum simulation: temporal evolution of the dimensionless instantaneous flow rate $Q_{i}^{2 D} /\left(\sqrt{g L^{3}}\right)$, as a function of the dimensionless time $t / \sqrt{L / g}$ for $L=90 d$ and $D=0.5 L$ (a) in 2D (b) in pseudo-3D with $W=22.5 d$. The dashed lines represent the mean flow rate $Q^{2 D} /\left(\sqrt{g L^{3}}\right)$.

granular flow is minimised. The computational cost is dominated by the solution of two Poisson-Helmholtz problems: a scalar Poisson equation for the pressure necessary to enforce incompressibility and a vector Poisson-Helmholtz equation for the time-implicit discretisation of the viscous term. Note that in contrast with the formulation in Gerris Lagrée et al. (2011), the full-coupled Poisson-Helmholtz problem for the velocity is solved (including coupling terms between velocity components). The simulations in this paper are in 2D. The CPU time of each simulation is always less than one hour on a laptop computer $\dagger$. We use a regularisation technique to avoid the divergence of the viscosity when the shear becomes too small by replacing $\eta$ by $\min \left(\eta, \eta_{\max }\right)$ with $\eta_{\max }=100 \mathrm{a}$ constant large enough, as done successfully in Lagrée et al. (2011) and Staron et al. (2012, 2014) where further details on the numerical method can be found.

\subsubsection{Pure 2D configuration}

We consider a two-dimensional silo of width $L$, along the $x$ axis, (gravity $\mathbf{g}$ is along negative $y$ axis) initially filled with a height $h_{p}=3.9 L$ of the visco-plastic fluid (see figure 1d). The mesh is such that the width of the silo $L$ is divided in 64 computation cells which is a good balance between precision and computational time.

We first performed a series of simulations in the two-dimensional case, imposing $\mathbf{f}_{w}=0$. We varied the size of the aperture $D$ and the particle diameters (see table 1). In figure $3 \mathrm{a}$, the instantaneous flow rate obtained for a given run, $Q_{i}^{2 D}$, is found to be close to stationary during the discharge as in the experiments. As done in the experiments, we measure the mean flow rate, $Q^{2 D}$, in the stationary regime (dashed lines in the figure).

\subsubsection{Averaged 2D or pseudo $3 D$ configuration}

To take into account the lateral friction on the walls and mimic 3D effects, we average the momentum equation across the width of the silo in the spirit of Hele-Shaw flows Jop et al. (2005); Lagrée (2007). For pure Hele-Shaw flows (with a Newtonian viscous fluid), the velocity is supposed to have a parabolic profile in the transverse $z$ direction. Here we

$\dagger$ The full code used is available and commented here:

http://basilisk.fr/sandbox/M1EMN/Exemples/granular_sandglass_muw.c 
suppose that the shape of the profile is almost flat, which reflects the yield-stress nature of the granular fluid. Hence the term appearing in front of the non-linear derivative term associated with the effective chosen profile is unity (see Lagrée 2007, for a discussion of the classical viscous case and bibliography). The integration of the viscous force across the cell gives the contribution of the friction stress at the wall, supposed to be a Coulomb force again: $-\mu_{w} p$ on each wall. This wall friction acts in the direction of the velocity. This gives the average additional force from the sidewalls in the momentum equation,

$$
\mathbf{f}_{w}=-2 \frac{\mu_{w} p}{W} \frac{\mathbf{u}}{|\mathbf{u}|} .
$$

From a Hele-Shaw point-of-view, the momentum 2.2 and incompressibility 2.1 equations apply to a $2 \mathrm{D}$-averaged velocity field $(u, v)$, with the extra source term in the momentum equation 2.2 taking into account the wall friction (2.4). We chose the value $\mu_{w}=0.1$ and varied the pseudo-width $W$, together with the aperture length $D$, and the particle diameter $d$, as shown in table 1 . Note that in the simulations $2 L \mu_{w} / W$ is the effective parameter. To compare with experiments and discrete dynamics (where the natural scale is $d$ ), the geometrical parameters of the continuous simulations are scaled by $d$ (see labels of figures 7 and 11).

In these pseudo-3D simulations, the different fields (velocity, pressure) are interpreted as width averages. The instantaneous flow rate is again found to be stationary during the discharge (figure $3 \mathrm{~b}$ ). We measured the mean flow rate $Q^{2 D}$ in the stationary regime (see the dashed lines in the figure) and we defined the equivalent 3D flow rate as $Q=W Q^{2 D}$.

Note that when the friction at the wall $\mathbf{f}_{w}$ were too large, the continuum simulations failed. We varied $2 L \mu_{w} / W$ up to about 5.6, but were not able to reach larger values. Further developments have to be done to overcome this problem, which could be related to the numerical method. Nonetheless the range of $W$ covered is sufficient to compare qualitatively the results of the simulations with the experiments.

\section{Effect of sidewalls on the silo discharge from a lateral orifice}

The aim of this study is to clarify the role of the two dimensions of the outlet, the height $D$ and the width $W$ as defined in figure 1, in the discharge of a silo from a lateral aperture. In a first part we have simplified the problem by considering a rectangular silo where the orifice spans the width of the silo. We first present the experimental results. We then discuss the role of the sidewalls on the flow rate using continuum simulations. Finally we extend the result to the cylindrical silo and we discuss the role of the particle diameter.

\section{1. $\Pi$-theorem}

The mass flow rate out of the silo, $Q$, depends on the density $\rho$, the gravity $g$ and the geometrical parameters: width $L$, orifice height $D$, thickness $W$, the filling height $h_{p}$ and the grain size $d$. Standard application of dimensional analysis or $\Pi$-theorem gives us the relation between non-dimensional numbers (eight quantities, three units, which gives five non-dimensional numbers). The flux must scale like $\rho \ell^{2} \sqrt{g \ell}$ where $\ell$ is any of $D, W, h_{p}, d$ (and $\ell^{2} \sqrt{\ell}$ any combination of these lengths), and this flux must also be a function of the geometric ratios e.g. $D / W, d / D, h_{p} / W, L / D$ (Note that if we use the mass of grains in the silo, this will give us an extra parameter with dimension, which can be reduced to a new dimensional number, the packing fraction $\phi_{b}$ ). Classical experiments on silos show that the flux is independent from the width $L$ (if large enough), from the filling height $h_{p}$ (if large enough), and from the grain size $d$ (if small enough). We can therefore 
$(a)$

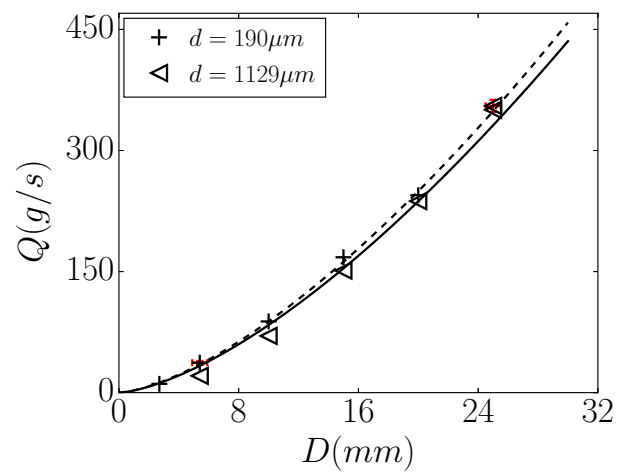

(b)

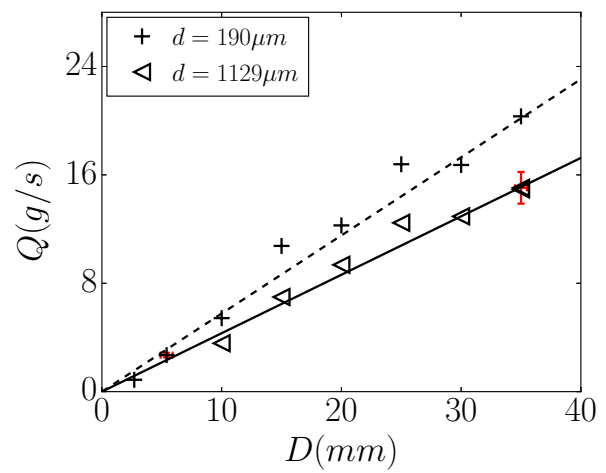

Figure 4: Experimental mass flow rate $Q$ in the rectangular silo versus the height of orifice $D$ for two sizes of particles $d$. (a) $W=40 \mathrm{~mm}$, the dashed (resp. full) line represents the equation $Q=c_{1} D^{3 / 2}$ where $c_{1}=2.79 \mathrm{~g} . \mathrm{s}^{-1} \mathrm{~mm}^{-3 / 2}\left(\right.$ resp. $\left.c_{1}=2.65 \mathrm{~g} . \mathrm{s}^{-1} \mathrm{~mm}^{-3 / 2}\right)$ is obtained using a least-squares fit. (b) $W=3.5 \mathrm{~mm}$, the dashed (resp. full) line represents the equation $Q=c_{2} D$ where $c_{2}=0.58$ g.s ${ }^{-1} \mathrm{~mm}^{-1}$ (resp. $c_{2}=0.43 \mathrm{~g} . \mathrm{s}^{-1} \mathrm{~mm}^{-1}$ ) is obtained using a least-squares fit.

neglect the corresponding geometric ratios. This then reduces $Q /\left(\rho \ell^{2} \sqrt{g \ell}\right)$ (where $\ell$ is any of $D, W)$ to a function of the aperture aspect ratio $\mathcal{A}=D / W$ only.

If $W$ is large, the problem becomes bidimensional and $D / W$ has no influence anymore. The velocity then scales like $\sqrt{g D}$, the size of the aperture being scaled by $D$, and the flux per transverse unit $Q / W$ is $\rho D \sqrt{g D}$. This gives the equivalent for this rectangular geometry of the Hagen-Berverloo relation (see a translation of the original article of Hagen in Tighe \& Sperl 2007; Beverloo et al. 1961):

$$
Q \propto W \rho \phi_{b} D \sqrt{g D}
$$

This scaling relation was recovered experimentally for a bottom aperture, spanning the width of a rectangular silo, by severals authors (Choi et al. 2005; Benyamine et al. 2014). This behaviour is also observed in our experimental set-up with a side aperture. It can be seen in figure $4 \mathrm{a}$ where the flow rate is plotted as a function of the outlet length $D$ for two particle diameters and for the larger silo thickness $W=40 \mathrm{~mm}$. For a given particle diameter the data are well described by equation 3.1, see the full line and the dashed line in the figure. Therefore, following the $\Pi$-theorem, the relevant normalisation for the flow rate seems to be $\rho \sqrt{g W^{5}}$, giving for the former relationship

$$
\frac{Q}{\rho \phi_{b} \sqrt{g W^{5}}}=c_{D}\left(\frac{D}{W}\right)^{3 / 2} .
$$

In the following section this scaling will be compared to the data obtained for the rectangular silo.

\subsection{Experimental results for the rectangular silo}

Figure 5a represents the dimensionless flow rate $Q_{\mathcal{A}}=Q /\left(\rho \phi_{b} \sqrt{g W^{5}}\right)$ versus the aperture aspect ratio $\mathcal{A}=D / W$, for various thicknesses $W$ and lengths $D$ of the orifice and for a given diameter of particle $d=190 \mu \mathrm{m}$. In this representation the data collapse as suggested by the $\Pi$-theorem. 
(a)

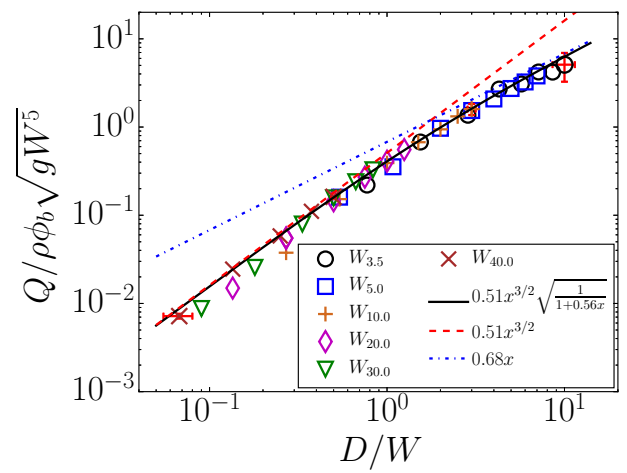

(b)

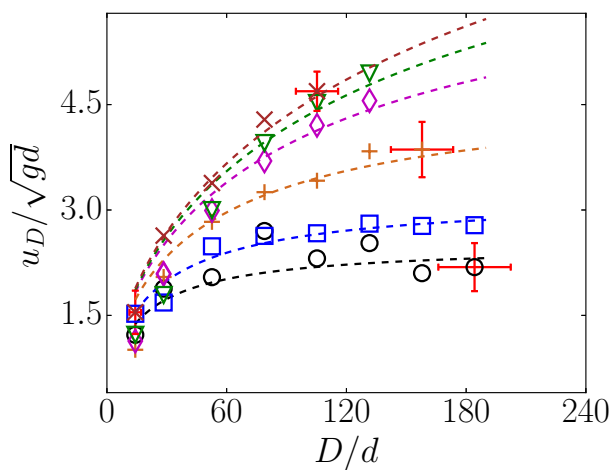

Figure 5: Experiments in the rectangular silo for a given particle diameter, $d=190 \mu \mathrm{m}$, and various silo widths, $W$. (a) dimensionless mass flow rate $Q /\left(\rho \phi_{b} \sqrt{g W^{5}}\right)$ as a function of $D / W$. The dashed line represents equation 3.2 with $c_{D}=0.51$, the dashed-dotted line represents equation 3.3 with $c_{W}=0.68$ and the solid line represents equation 3.4 with the same parameters. (b) Corresponding dimensionless discharge velocity $u_{D} / \sqrt{g d}$ as a function of $D / d$. The dashed lines represent equation 3.5 with the same parameters.

As expected, for large thicknesses $(D / W \ll 1), Q_{\mathcal{A}}$ follows a power law with an exponent $3 / 2$ corresponding to the Hagen-Beverloo relation (equation 3.2), see the dashed line in the figure. However, for large values of $D / W$ (i.e. thin-enough silos), an important experimental result is that the dimensionless flow rate of particles depends linearly on the aperture aspect ratio (see the dashed-dotted line in figure 5a):

$$
\frac{Q}{\rho \phi_{b} \sqrt{g W^{5}}}=c_{W} \frac{D}{W} .
$$

This can also be seen in figure $4 \mathrm{~b}$ where the flow rate is plotted versus the aperture length $D$ for the smallest thickness explored $W=3.5 \mathrm{~mm}$ and for two particles diameters $d$. For a given particle diameter the flow rate indeed exhibits a linear trend $Q \propto D$. The transition between these two regimes occurs around a specific aperture ratio $\mathcal{A}_{c} \approx 2$. According to these two asymptotic regimes, we can thus propose a fitting formula for the flow rate:

$$
\frac{Q}{\rho \phi_{b} \sqrt{g W^{5}}}=c_{D}\left(\frac{D}{W}\right)^{3 / 2} \frac{1}{\sqrt{1+\left(c_{D} / c_{W}\right)^{2} D / W}} .
$$

As illustrated in figure $5 \mathrm{a}$ (solid lines), this formula describes well the flow rate dependency on aperture dimensions in any regime, with the fitting parameters $c_{D}=0.51$ and $c_{W}=0.68$.

These two regimes can be interpreted in term of the (horizontal) discharge velocity $u_{D}=Q / \rho \phi_{b} W D$, which can be expressed using equation 3.4 as

$$
u_{D}=c_{D} \sqrt{g D} \frac{1}{\sqrt{1+\left(c_{D} / c_{W}\right)^{2} D / W}} .
$$

This relation again describes the data very well whatever the silo width as can be seen in figure $5 \mathrm{~b}$ (dashed lines). The first regime corresponds to the classical Hagen-Beverloo relation with a velocity which scales with the aperture length:

$$
(D / W)<\mathcal{A}_{c}, \quad u_{D}=c_{D} \sqrt{g D} .
$$


(a)

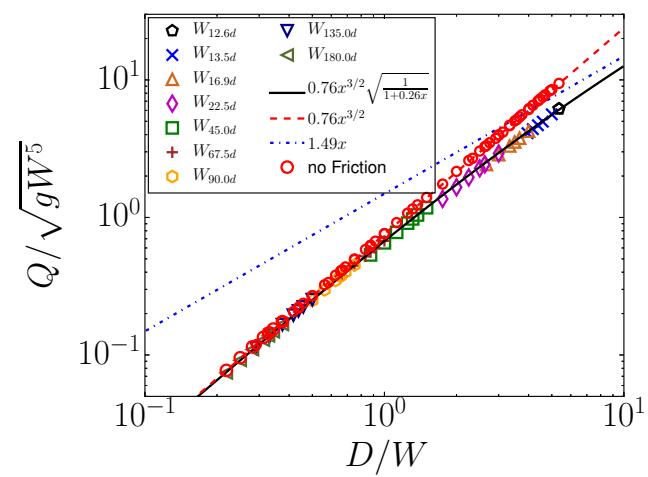

(b)

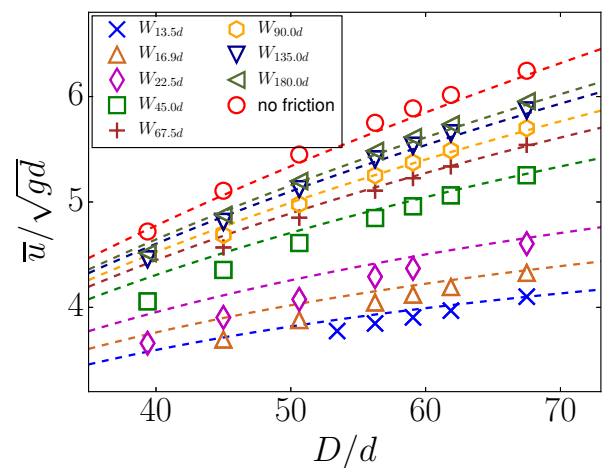

Figure 6: Continuum simulations results in 2D and in pseudo-3D for various $W$ with $H=360 d, L=90 d$ and $D=45 d$ : (a) Flow rate $Q$ normalized by $\sqrt{g W^{5}}$ as a function of $D / W$. The dashed line represents equation 3.2 with $c_{D}=0.76$, the dashed-dotted line represents equation 3.3 , with $c_{W}=1.49$, and the solid line represents equation 3.4 , with the same parameters. (b) Mean horizontal velocity at the orifice $\bar{u} / \sqrt{g d}$, as a function of $D / d$. The dashed lines represent equation 3.5 , with the same parameters.

Whereas in the second regime the discharge velocity scales with the aperture width:

$$
(D / W)>\mathcal{A}_{c}, \quad u_{D}=c_{W} \sqrt{g W} .
$$

This gives a velocity scaling with the smallest of the two lengths $W$ and $D$.

Historically the Hagen-Beverloo relation was explained with the concept of a "free-fall arch" located at the outlet, from which the particles fall freely. Several recent experiments and numerical simulations question this hypothesis. From an experimental point of view, Janda et al. (2012); Rubio-Largo et al. (2015) showed that the granular medium remains dense, with a small dilation, close to the outlet, and that the particles do not undergo a free fall. These observations were validated using discrete simulation (Rubio-Largo et al. 2015). Moreover Navier-Stokes simulations, assuming a continuum frictional rheology of the granular media, have been successfully used to recover the Hagen-Beverloo scaling as in Staron et al. (2012, 2014); Davier \& Bertails-Descoubes (2016); Dunatunga \& Kamrin (2015), with very different numerical methods.

The fact that we recover the Hagen-Beverloo relation with a vertical aperture also tends to refute this concept, as pointed out by Sheldon \& Durian (2010). Based on these observations, we performed a continuum simulation, using our Navier-Stokes solver with the granular rheology, to test whether we can reproduce at least qualitatively the experimental behaviour.

\subsection{Comparison with continuum numerical simulations}

We first performed continuum numerical simulations in the $2 \mathrm{D}$ case. To compare with the experimental results we have plotted in figure 6 a the dimensionless flow rate $Q_{\mathcal{A}}=$ $Q /\left(\sqrt{g W^{5}}\right)$ versus the aperture aspect ratio $\mathcal{A}=D / W$, using the same series of data but rescaled several times with each width $W$ used for the pseudo-3D simulations. In a pure bidimensional flow (red circles), the Navier-Stokes simulations recover the HagenBeverloo scaling, equation 3.2 (dashed line). This suggests that the $\mu(I)$ fluid rheology provides consistent results for the discharge of a silo with a side-located aperture.

Then, to mimic the effects of the lateral walls from a Hele-Shaw point-of-view, we 
added the friction term proportional to the pressure and aligned with the velocity, equation 2.4, and we varied the pseudo-width $W$ (see the different symbols in figure 6a). We first notice that when the aperture ratio is small $\mathcal{A}=D / W$, similar to the experimental case, the data of the pseudo-3D simulations are superimposed onto the $2 \mathrm{D}$ simulations, and we recover the Hagen-Beverloo scaling, equation 3.2. When increasing the aperture ratio, we observe a departure from this scaling towards a regime where $Q_{\mathcal{A}} \propto \mathcal{A}$ (see the dashed-dotted line). We can see that we do not completely reach this regime due to the numerical upper limit of achievable values for $\mathbf{f}_{w}$, however the data are well described by equation 3.4 with $c_{D}=0.76$ and $c_{W}=1.49$ (solid line).

Following the experimental section, the dimensionless mean horizontal velocity at the outlet, $\bar{u} / \sqrt{d g}$, is plotted versus the dimensionless outlet length $D / d$ in figure $6 \mathrm{~b}$. We observe the same trends as for the experimental data: in the regime controlled by the outlet length $D$, the velocity tends toward the Hagen-Beverloo scaling (equation 3.6 and $2 \mathrm{D}$ data) whereas when the ratio $\mathcal{A}$ increases, the velocity tends toward the regime controlled by the silo width given by equation 3.7. Again each series of data for a given $W$ are fairly well described by equation 3.5 (dashed lines).

In the continuum simulation, the width of the silo appears only in the term describing the sidewall friction, which suggests that the second regime is controlled by this term. It is interesting to note that in the regime dominated by the lateral friction the flow rate per unit length at a given $D$ is lower than in the first regime. Even if we cannot fully reach the second regime $(\mathcal{A} \gg 1)$, we carry on with the analysis and present some comparisons of the details of the internal fields at the limit of the numerical model.

Figure 7 shows the velocity field and the streamlines of both the experimental and the numerical runs for increasing silo width. The numerical velocity field (figure $7 \mathrm{~b}$ ) is qualitatively very similar to the experimental velocity field (figure 7a). In both configurations, the streamlines tend to vertical lines upward from the orifice at a distance which decreases when $W$ increases. On the same figures, we observe that the flowing zone is found to be thinner when the lateral friction increases (i.e. when $W$ decreases). The limit of the stagnant zone for various outlet sizes $D$ is plotted in figure 8 both for the experiment (a), and the continuum simulation (b), in the regime dominated by the lateral friction. Interestingly this position does not depend on $D$ in either cases, but depends strongly on $W$.

We may explain these behaviours at small $W$ by looking at the Navier-Stokes equations. Let us consider a steady flow (implying that we neglect inertia). As the friction at the walls increases when $W$ decreases, the friction term (of magnitude $\mu_{w} p / W$ ) will be at most as large as gravity $(\rho g)$. The gradients of the stress tensor are of order $\mu_{s} p / L$. This order of magnitude may be rewritten as $\left(\mu_{s} / \mu_{w}\right)(W / L) \mu_{w} p / W$. It is clearly smaller than $\mu_{w} p / W$ as $\left(\mu_{s} / \mu_{w}\right)=O(1)$ and $(W / L) \ll 1$. Hence, as the wall friction increases to balance gravity, the momentum equation can be approximated as:

$$
0 \simeq \frac{2 \mu_{w} p}{W} O\left(\frac{\mu_{s} W}{\mu_{w} L}\right) \boldsymbol{e}+\rho \mathbf{g}-\frac{2 \mu_{w} p}{W} \frac{\mathbf{u}}{|\mathbf{u}|},
$$

where $\boldsymbol{e}$ is a unit vector in the main direction of the force resulting from internal friction. The velocity $\mathbf{u}$ is aligned with gravity at first order, the more so as the thickness decreases. This is noticeable in figure $7(\mathrm{c}, \mathrm{d})$ where for small $W$ the streamlines are clearly more vertical than for larger $W$. To quantify this effect, we have plotted the angle of inclination $\theta$ of the central streamline at the orifice relative to the vertical in figure 9 . If we note $u_{0}$ the horizontal velocity at the center of the outlet, and $U_{0}$ the norm of the velocity at this position, we can write $\sin \theta=u_{0} / U_{0}$ (note that $u_{0}$ is proportional to the previously defined discharge velocity $u_{D}$, as we will see in section A.2). We observe in figure 9 a that 
(a)

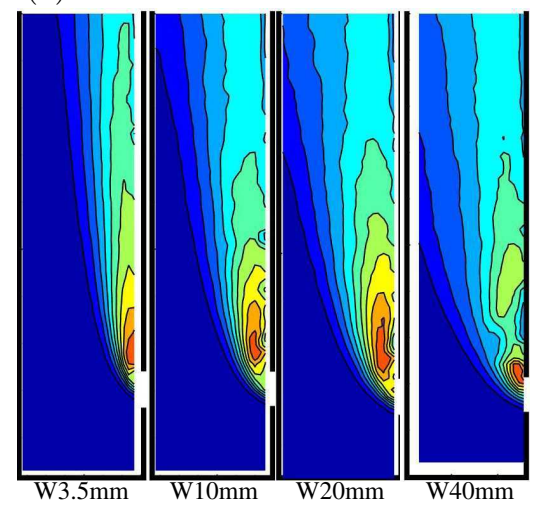

(c)

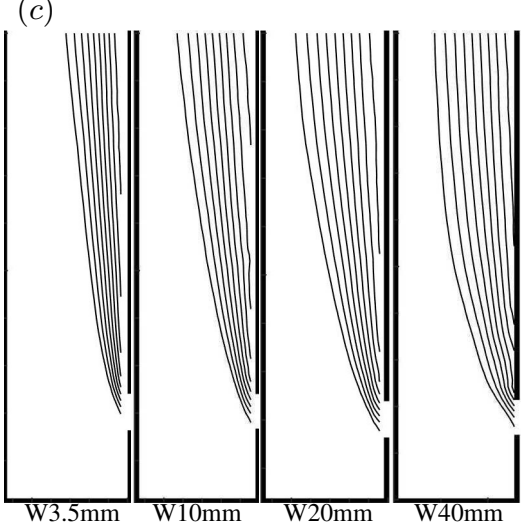

(b)

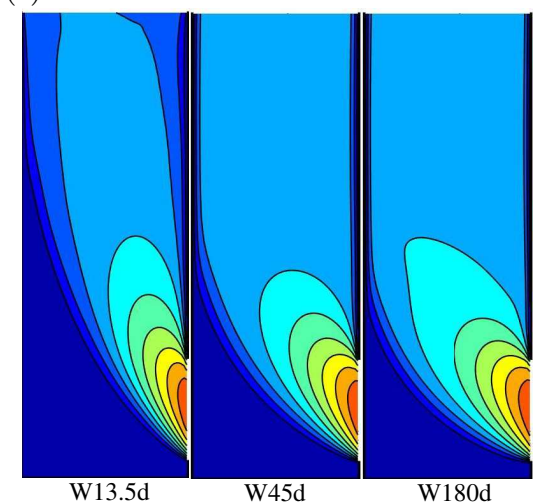

(d)

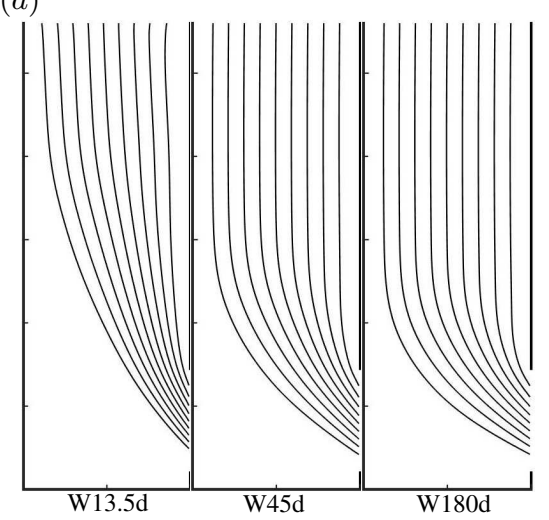

Figure 7: Velocity field (a,b) and streamlines (c,d) for the experiment (a,c) with $D=$ $20 \mathrm{~mm}, d=1129 \mu \mathrm{m}$ and $W=[3.5,10,20,40] \mathrm{mm}$, and for the continuum simulation $(\mathrm{b}, \mathrm{d})$ with $D=56.25 d$ and $W=[13.5 d, 45 d, 180 d]$.

(a)

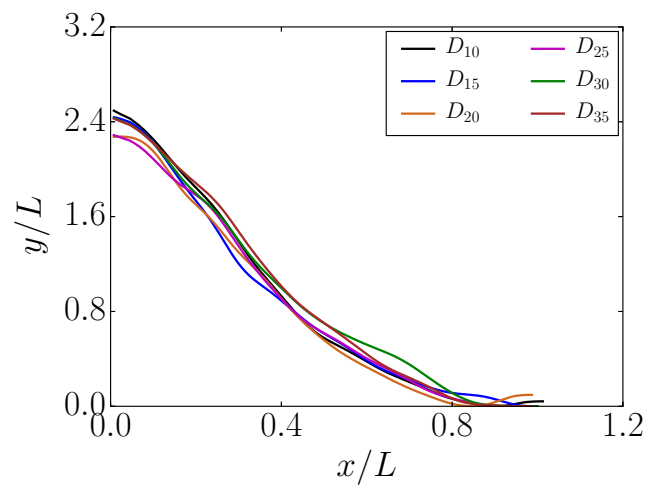

(b)

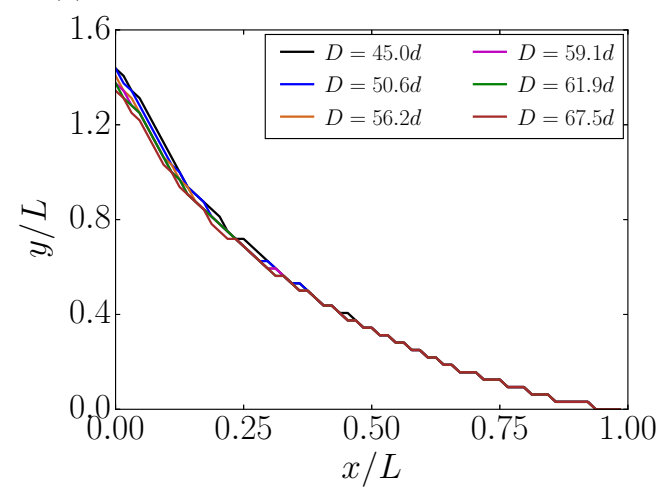

Figure 8: Limit of the stagnant zone for various $D$ (a) experiments with $W=5 \mathrm{~mm}$ and (b) continuum simulations with $W=17.4 d$. 

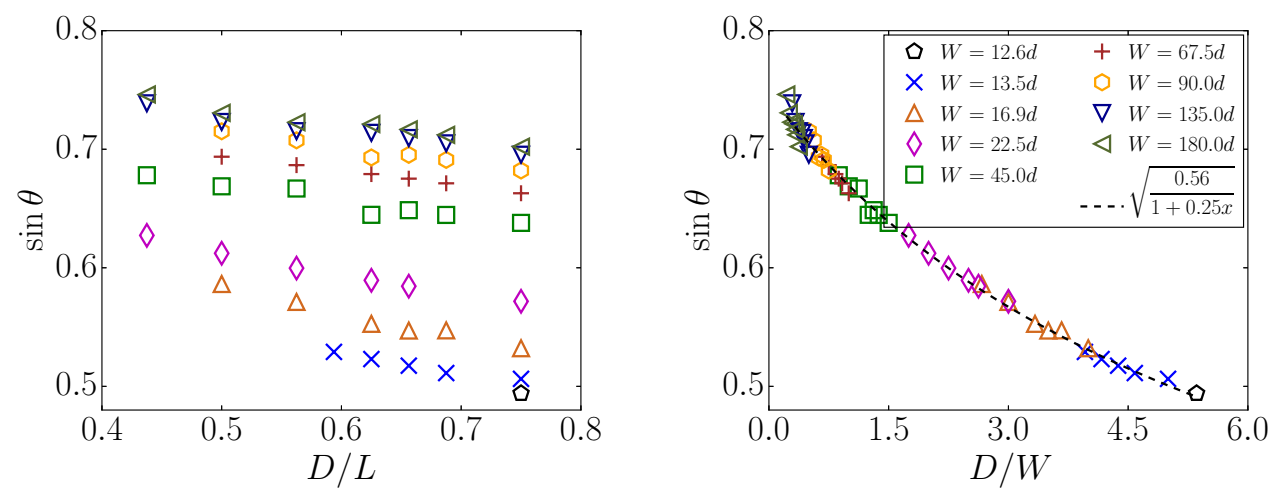

Figure 9: Continuum simulation results: angle of inclination $\theta$ of the central streamline at the orifice relative to vertical for various $W$ (a) versus $D / L$, (b) versus $D / W$. The dashed line represents equation 3.10 with $\gamma_{1}=0.56$ and $\gamma_{2}=0.25$.

this angle decreases slightly with $D$, and strongly with $W$ as expected. More interestingly, if plotted versus $D / W$ (figure $9 \mathrm{~b}$ ), the data superimpose. In order to study the scaling relation of $\sin \theta$, we then turn to the dependency of the norm of the velocity $U_{0}$ and horizontal velocity $u_{0}$ on $W$ and $D$. In figure 10 we have represented the norm of the velocity $U_{0} / \sqrt{g d}$ on the central streamline at the orifice versus $D / d$ for various $W$. Surprisingly we can see that the norm of the velocity does not significantly depend on $W$. The data can be described by

$$
U_{0}=c_{U} \sqrt{g D}
$$

with $c_{U}=1.2$. This suggests that the kinetic energy always scales like $\rho g D$ whatever the lateral friction. For the horizontal velocity $u_{0}$, since the flow rate $Q \propto u_{0} W D$, from equation 3.4 , we can write

$$
u_{0} \propto \sqrt{g D} \sqrt{\frac{1}{1+\left(c_{D} / c_{W}\right)^{2} D / W}} .
$$

We can thus obtain

$$
\sin \theta=\sqrt{\frac{\gamma_{1}}{1+\gamma_{2} D / W}} .
$$

Moreover we see that the data in figure $9 \mathrm{~b}$ is well described by this formulation with $\gamma_{1}=$ 0.56 and $\gamma_{2}=0.25$, with the fitting parameter $\gamma_{2}=0.25$ corresponding to $\left(c_{D} / c_{W}\right)^{2}=$ 0.26 .

Finally figure 11 shows the time evolution of the top surface of granular material for various silo widths for (a) the experiment and (b) the continuum simulation. From experiments we can see that the surface of particles begins to tilt in the early stage for a small width $W$, whereas it remains constant till the last stage for the largest $W$. The tilted interface exhibits a slope starting from the wall opposite to the outlet and reaches a flattened surface, or sometimes even a small bump, on the wall containing the outlet. The slope of the interface is higher than the angle of repose for $W=3.5 \mathrm{~mm}$, and smaller than the angle of repose for $W=10 \mathrm{~mm}$. In continuum simulations, we explored a smaller range of $W$, the smallest $W$ case $W=13.5 d$, is comparable to the case $W=20 \mathrm{~mm}$ in the experiments, but we clearly recover the same trend: the larger the thickness $W$, the longer the interface remains symmetrical. Note also that the no-slip 


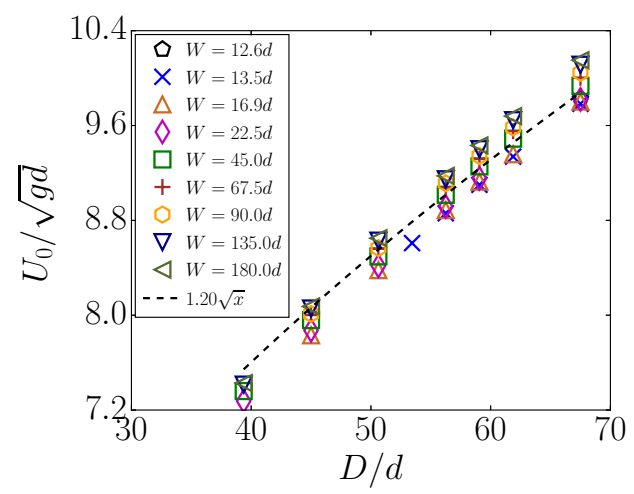

Figure 10: Continuum simulation results: norm of the velocity $U_{0} / \sqrt{g d}$ on the central streamline at the orifice versus $D / d$ for various $W$. The dashed line represents equation 3.8 with $c_{U}=1.2$.

(a)

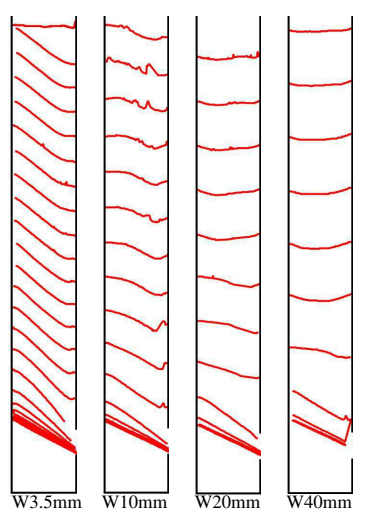

(b)

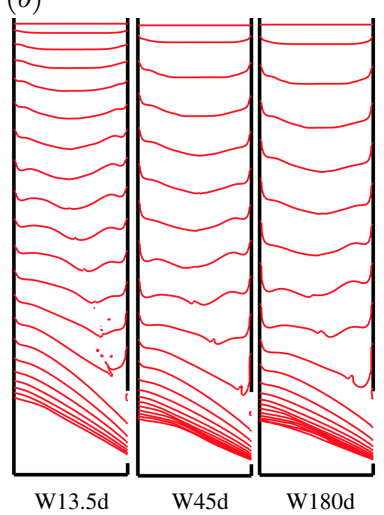

Figure 11: Profiles of the top surface of granular materials for various $W$ at constant time steps: (a) experiments with $D=25 \mathrm{~mm}, d=190 \mu \mathrm{m}$, time interval $\Delta t_{e}=0.5 \mathrm{~s}$, (b) continuum simulation with $D=56.25 d$ and time interval $\Delta t_{s} / \sqrt{d / g}=9.5$.

conditions imposed at the wall are not exactly the same as in the experiments, where a sliding velocity is observed. Nevertheless, the same qualitative profiles are obtained. This behaviour is consistent with the previous observation: for large $W$, the flow far from the outlet is symmetrical. For small $W$, the lateral friction breaks this symmetry and localises the flow on the side of the outlet, which inclines the surface in this direction.

\subsection{Experimental results in the cylindrical silo}

The previous results were given for a simplified geometry, with a rectangular silo and an outlet which spans the width of the silo. Experimentally we also performed measurements of the flow rate for a cylindrical silo, with an outlet located at its side as schematised in figure $1 \mathrm{~b}$. This situation generates a flow in a fully $3 \mathrm{D}$ geometry, and is of practical interest. To characterise the different roles played by the length $D$ and the width $W$ of the outlet, we have plotted in figure 12 the dimensionless mass flow rate $Q /\left(\rho \phi_{b} \sqrt{g W^{5}}\right)$ as a function of the aperture aspect ratio $D / W$, as done in the rectangular configuration.

As suggested by the $\Pi$-theorem in section 3.1 the data superimpose in this representa- 


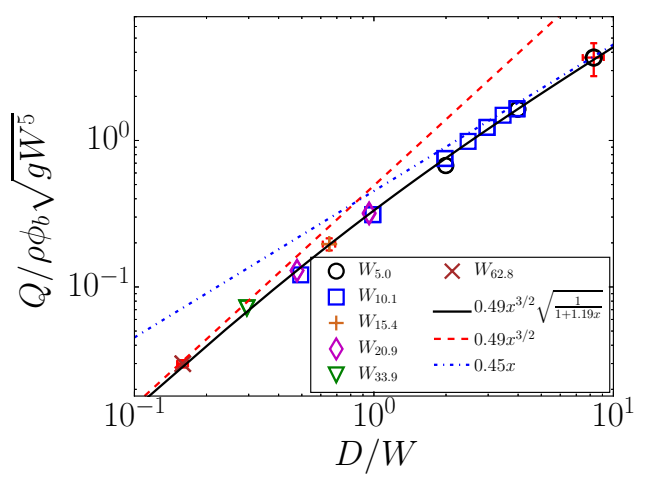

Figure 12: Experimental results in the cylindrical silo: dimensionless mass flow rate $Q /\left(\rho \phi_{b} \sqrt{g W^{5}}\right)$ as a function of the aperture aspect ratio $D / W$. The dashed line represents equation 3.2 with $c_{D}=0.49$, and the dashed-dotted line represents equation 3.3 with $c_{W}=0.46$.

tion. Moreover, we observe the same behaviours as in rectangular silos. For large $W$, the flow rate follows the Hagen-Beverloo relation (equation 3.2, dashed line in the figure) and for small $W$, the flow rate follows equation 3.3 (dashed-dotted line in the figure) suggesting that the flow is dominated by the lateral friction. Over the whole range, the data are well correlated by equation 3.4 (solid line in the figure) with $c_{D}=0.49$ and $c_{W}=0.46$. The fitting parameter $c_{w}$ is found to be slightly smaller than that in the rectangular silo. This suggests that the dissipation is higher in the cylinder, and more specifically in the regime controlled by the lateral friction where we can assume that the friction with a smooth lateral wall is smaller than that with an erodible granular media. Consequently, the transition between the two regimes occurs for a smaller aperture aspect ratio $\mathcal{A}_{c} \approx 1$.

\subsection{Dependency of the flow rate on the particle diameter}

The previous results are shown for a given small particle diameter $d=190 \mu \mathrm{m}$. In this section we focus on the influence of the particle diameter. We have shown that the flow rate of discharge of a silo with a lateral outlet depends on the aperture aspect ratio $\mathcal{A}=D / W$, and exhibits two regimes of flow described by equation 3.2 for small $\mathcal{A}$ and by equation 3.3 for large $\mathcal{A}$. When the particle diameter is varied, these scaling relations seem to remain valid, as can been seen in figures $4(\mathrm{a}, \mathrm{b})$ where the flow rate is plotted versus $D$ for two particle diameters, $d=190 \mu \mathrm{m}$ and $d=1129 \mu \mathrm{m}$, in the two regimes. In each regime the same scaling is found for the two grain sizes, however we observe a shift of these relations towards smaller flow rates when the particle diameter increases. This behaviour can be seen in figure 13(a,c) where the dimensionless flow rate $Q_{\mathcal{A}}=Q / \rho \phi_{b} \sqrt{g W^{5}}$ is plotted as a function of $D / W$ for both the rectangular silos and the cylindrical silo for all the batch of particles we used. The two regimes are recovered whatever the particle diameter, see the dashed line and the dashed-dotted line. However there is a significant scattering of the data above the relation fitted for the smallest particle size $d=190 \mu \mathrm{m}$.

To model the dependency of the flow rate on the particle diameter, we follow the work of Janda et al. (2012). In this framework we suppose that the particle size has to be accounted for through the number of beads in the aperture via a geometrical function, 
(a)

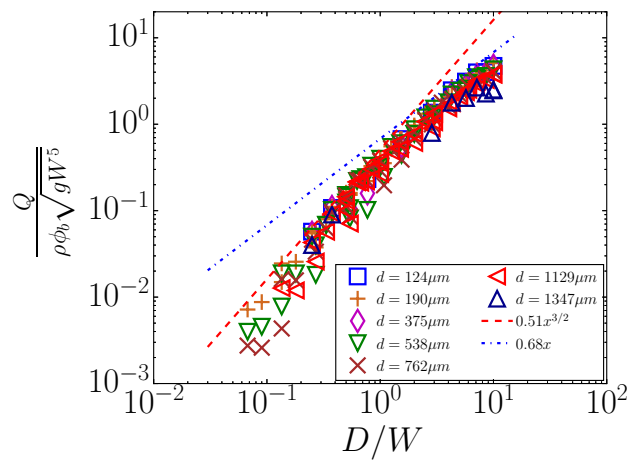

(c)

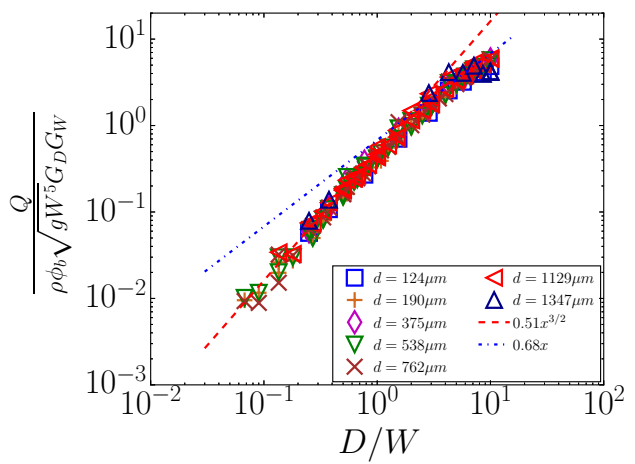

(b)

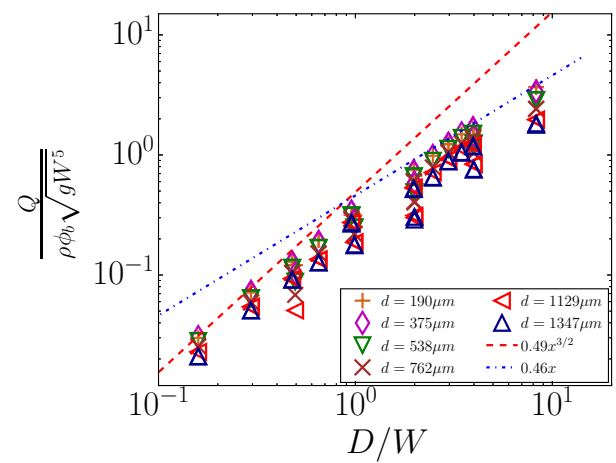

$(d)$

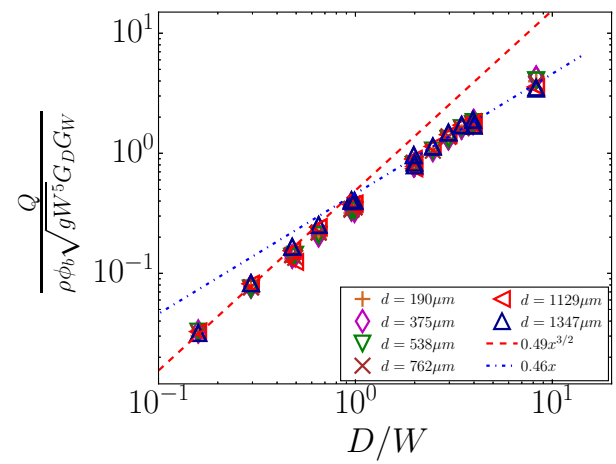

Figure 13: Mass flow rate $Q$ as a function of $D / W$ for various $d$ for (a,c) rectangular silos and (b,d) cylindrical silos, normalized in $(\mathrm{a}, \mathrm{b})$ by $\left(\rho \phi_{b} \sqrt{g W^{5}}\right)$ and in $(\mathrm{c}, \mathrm{d})$ by $\rho \phi_{b} \sqrt{g W^{5}} G_{D}(D / d) G_{W}(W / d)$ (see equation 3.11) with the fitting parameters $\beta_{D}=$ $\beta_{W}=0.1, \alpha_{D}=1$ and $\alpha_{W}=0.46$ (see appendix A). The dashed and dashed-dotted lines are the same as in figure 5 and 12.

significant when this number is small. We take the two dimensions of the aperture into account and thus we suppose that the flow rate depends on two geometrical functions based on the number of beads in the aperture length $D / d$, and in the aperture width $W / d$. The details of this approach and its validation using a $2 \mathrm{D}$ discrete numerical simulation are given in the appendix. This yields the following expression for the flow rate:

$$
Q=C_{l}^{\prime} \rho \phi_{b} G_{D}\left(\frac{D}{d}\right) G_{W}\left(\frac{W}{d}\right) F\left(\frac{D}{W}\right) W D \sqrt{g D},
$$

with $G_{D}(D / d)=\left[1-\alpha_{D} e^{-\beta_{D} \frac{D}{d}}\right], G_{W}(W / d)=\left[1-\alpha_{W} e^{-\beta_{W} \frac{W}{d}}\right]$ and $F(D / W)=$ $\sqrt{1 /\left[1+\gamma_{2} D / W\right]}$.

Figure 13 shows the normalised flow rate using the geometrical functions, $Q /\left[\rho \phi_{b} G_{D}(D / d) G_{W}(W / d) \sqrt{g W^{5}}\right]$, as a function of the aperture aspect ratio $D / W$ for the whole range of particle diameters for (c) rectangular silos and (d) cylindrical silos. In this representation the data collapse and can be well adjusted by the same asymptotic relations in two regimes as in figure 5 and 12.

We propose additional analysis in the appendix. The $2 \mathrm{D}$ discrete numerical simulation 
shows that the velocity at the outlet may follow the same geometrical relation than the volume fraction. As the continuum simulation described correctly most of the observations on the discharge of a silo with a lateral aperture for a given particle diameter we wonder if the continuum simulation is able to recover partially the dependency on the particle size of the flow rate. Indeed, the $\mu(I)$ rheology contains the information on the particle diameter in the definition of the inertial number $I=d \sqrt{2} D_{2} /(\sqrt{p / \rho})$. Even though a dependency on the particle diameter is observed in the $2 \mathrm{D}$ case, the profiles present a larger asymmetry than the one predicted by the $2 \mathrm{D}$ discrete simulations. This can be due to the fact that the volume fraction has been assumed to be uniform, whereas the complete $\mu(I)$-rheology, that includes a $\phi(I)$ relation, would predict a dilation of the granula medium when it is sheared.

\section{Conclusion and perspectives}

Using experiments, 2D discrete simulations and continuum simulations we have studied the discharge of a silo with a lateral orifice. Experimentally we have observed two regimes of flow, either in a rectangular silo with an orifice spanning the thickness $W$ of the silo, or in a cylindrical silo. The first regime, observed for small aperture aspect ratios $\mathcal{A}=D / W$, corresponds to the well known Hagen-Beverloo regime with a flow rate $Q \propto W D^{3 / 2}$. The second regime, observed for large aperture aspect ratios $\mathcal{A}>\mathcal{A}_{c}$, follows $Q \propto W^{3 / 2} D$. We have proposed an empirical relation which predicts the flow rate depending on the aperture dimensions and which recovers both regimes. The continuum simulation, using the frictional $\mu(I)$ rheology and solved in $2 \mathrm{D}$ with an additional force to take into account the wall friction in a Hele-Shaw spirit, is found to describe qualitatively these two regimes and most of the internal details of the flow field observed experimentally in the rectangular silo, notably the fact that the velocity field is aligned with gravity when lateral wall friction is large. The dependency on the silo width suggests that the large $\mathcal{A}$ regime is dominated by the lateral friction, and it seems reasonable to think that the small $\mathcal{A}$ regime is controlled by the internal friction.

We have found that the particle diameter matters only when the number of beads through the smallest orifice dimension is sufficiently small. The flow rate dependency on this parameter can be reasonably described using two geometrical functions $G_{\ell}(\ell / d)=$ $1-\alpha e^{-\beta \ell / d}$ based respectively on $W$ and on $D$. A large part of this behaviour is well reproduced using $2 \mathrm{D}$ discrete simulations. Interestingly a dependency on the particle diameter is also observed in the $2 \mathrm{D}$ continuum simulation but it predicts different velocity profiles which can be due to the fact that in the continuum simulation the volume fraction does not vary.

In future work these promising observations need to be confirmed by conducting systematic 3D simulations. Preliminary 3D continuum simulation results indicate that the Hagen-Beverloo relation is obtained for a silo with bottom aperture. Extra developments have to be done to include realistic boundary conditions to represent particles-wall friction as well as the volume fraction variation $\phi(I)$.

\section{Acknowledgements}

We would like to thank P. Cervetti, S. Noel and F. Ratouchniak for technical assistance. This work has been carried out in the framework of the Labex MEC (ANR10-LABX-0092) and of the A*MIDEX project (ANR-11-IDEX-0001-02), funded by the 'Investissements d'Avenir' French Government program managed by the French National Research Agency (ANR). 


\section{REFERENCES}

Aguirre, M. A., Grande, J. G., Calvo, A., Pugnaloni, L. A. \& Géminard, J.-C. 2010 Pressure independence of granular flow through an aperture. Phys. Rev. Lett. 104, 238002.

Benyamine, M., Duermane., M., Dalloz-Dubrujeaud, B. \& Aussillous, P. 2014 Discharge flow of a bidisperse granular media from a silo. Phys. Rev. E 90, 032201.

Bertho, Y., Giorgiutti-Dauphiné, F. \& Hulin, J.-P. 2003 Dynamical Janssen effect on granular packing with moving walls. Phys. Rev. Lett. 90 (14), 144301.

Beverloo, W. A., Leniger, H. A. \& Van De Velde, J. 1961 The flow of granular solids through orifices. Chem. Eng. Sci. 15, 260-269.

Choi, J., Kudrolli, A. \& Bazant, M. Z. 2005 Velocity profile of granular flows inside silos and hoppers. J. Phys.: Condens. Matter 17, S2533-S2548.

Davier, G. \& Bertails-Descoubes, F. 2016 Nonsmooth simulation of dense granular flows with pressure-dependent yield stress. J. Non-Newton. Fluid 234, 15-35.

Dunatunga, S. \& Kamrin, K. 2015 Continuum modelling and simulation of granular flows through their many phases. J. Fluid Mech. 779, 483-513.

JAndA, A., Zuriguel, I. \& MAZA, D. 2012 Flow rate of particles through apertures obtained from self-similar density and velocity profiles. Phys. Rev. Lett. 108, 248001.

Jop, P., Forterre, Y. \& Pouliquen, O. 2005 Crucial role of sidewalls in granular surface flows: consequences for the rheology. J. Fluid Mech. 541, 167-192.

Jop, P., Forterre, Y. \& Pouliquen, O. 2006 A constitutive law for dense granular flows. Nature 441, 727-730.

Lagrée, P.-Y. 2007 Interactive boundary layer in a Hele Shaw cell. Z. Angew. Math. Mech. 87 (7), 489-498.

Lagrée, P.-Y., Staron, L. \& Popinet, S. 2011 The granular column collapse as a continuum: validity of a Navier-Stokes model with a $\mu(I)$-rheology. J. Fluid Mech. 686, 378-408.

Medina, A., Cabrera, D., López-Villa, A. \& Pliego, M. 2014 Discharge rates of dry granular material from bins with lateral exit holes. Powder Technol. 253, 270-275.

Meunier, P. \& Leweke, T. 2003 Analysis and treatment of errors due to high velocity gradients in particle image velocimetry. Exp. Fluids 35 (5), 408-421.

Midi, G. D. R. 2004 On dense granular flows. Eur. Phys. J. E 14 (4), 341-365.

Perge, C., Aguirre, M.A., Gago, P.A., Pugnaloni, L.A., Tourneau, D. Le \& Géminard, J.C. 2012 Evolution of pressure profiles during the discharge of a silo. Phys. Rev. E Stat. Nonlin. Soft Matter Phys. 85.

Popinet, S. 2003 Gerris: a tree-based adaptive solver for the incompressible euler equations in complex geometries. J. Comput. Phys. 190 (2), $572-600$.

Popinet, S. 2009 An accurate adaptive solver for surface-tension-driven interfacial flows. Journal of Computational Physics 228 (16), 5838-5866.

Popinet, S. 2013-2016 Basilisk C reference manual. http://basilisk.fr/Basilisk C.

Radjai, F. \& Dubois, F. 2011 Discrete-element modeling of granular materials. Wiley-Iste.

Rubio-Largo, S.M., Janda, A., Maza, D., Zuriguel, I. \& Hidalgo, R.C. 2015 Disentangling the free-fall arch paradox in silo discharge. Phys. Rev. Lett. 114, 238002.

Serrano, D.A., Medina, A., Chavarria, G. Ruiz, Pliego, M. \& Klapp, J. 2015 Mass flow rate of granular material flowing from tilted bins. Powder Technol. 286, 438-443.

Sheldon, H. G. \& Durian, D. J. 2010 Granular discharge and clogging for tilted hoppers. Granul. Matter 12, 579-585.

Staron, L., Lagrée, P.-Y. \& Popinet, S. 2012 The granular silo as a continuum plastic flow: The hour-glass vs the clepsydra. Phys. Fluids 24, 103301.

Staron, L., Lagrée, P.-Y. \& Popinet, S. 2014 Continuum simulation of the discharge of the granular silo, a validation test for the $\mu(\mathrm{I})$ visco-plastic flow law. Eur. Phys. J. E 37 (5).

Tighe, B.P. \& Sperl, M. 2007 Pressure and motion of dry sand: translation of Hagen's paper from 1852. Granul. Matter 9, $141-144$.

Zhou, Y., Ruyer, P. \& Aussillous, P. 2015 Discharge flow of a bidisperse granular media from a silo: discrete particle simulations. Phys. Rev. E 92, 062204. 


\section{Appendix A. Additional analysis}

In this section we focus on the influence of the particle diameter. We first present an analysis of the experimental results based on the work of Janda et al. (2012). We then discuss the role of the particle diameter on the flow close to the outlet using 2D Contact Dynamics simulations. Finally, we compare these results to the continuum numerical simulations.

\section{A.1. Experimental results and discussion}

To take the dependency on the particle diameter into account, as observed in figure 13(a,b), we suppose that the observations made experimentally by Janda et al. (2012), and numerically by Zhou et al. (2015) for a two-dimensional silo with an orifice placed at the bottom of the silo remain valid in our geometry. We make the following assumptions:

(i) We suppose that the horizontal velocity profile together with the density profile at the outlet are self-similar when varying the length of the hole $R=D / 2$. The flow rate is then given by

$$
Q=\rho W \iint_{-R}^{R} \phi(y) u(y) d y=c \rho W D \bar{\phi} \bar{u},
$$

where $c$ is a constant of integration and $\bar{\phi}$ and $\bar{u}$ represent the mean density and the mean horizontal velocity at the outlet respectively. The flow rate is then determined by these two quantities.

(ii) Following equation 3.5, the mean horizontal velocity tends asymptotically as $d$ decreases towards

$$
\xi_{v} \sqrt{g D} \sqrt{\frac{1}{1+\gamma_{2} D / W}}=\xi_{v} \sqrt{g D} F(D / W),
$$

where $F(D / W)=\sqrt{1 /\left[1+\gamma_{2} D / W\right]}$, which corresponds to the value for infinitely-small particles.

(iii) The granular medium tends to dilate at the outlet to maintain the flow. However for a large number of beads in the aperture, we suppose that it tends towards a fraction of the bulk density asymptotically, $\xi_{\phi} \phi_{b}$, with $\xi_{\phi}$ a constant.

(iv) We suppose that both the mean density and the mean velocity depend on the number of beads in the aperture via a geometrical function. To take the two dimensions of the aperture into account, we suppose that the flow rate depends on two geometrical functions based on the number of beads in the aperture length $D / d$, and in the aperture width $W / d$. We assume that this geometrical function can be fitted by an exponential saturation

$$
Q=C_{l}^{\prime} \rho \phi_{b} G_{D}\left(\frac{D}{d}\right) G_{W}\left(\frac{W}{d}\right) F\left(\frac{D}{W}\right) W D \sqrt{g D}
$$

with $G_{D}(D / d)=\left[1-\alpha_{D} e^{-\beta_{D} \frac{D}{d}}\right]$ and $G_{W}(W / d)=\left[1-\alpha_{W} e^{-\beta_{W} \frac{W}{d}}\right]$.

To test these hypotheses, we isolated each geometrical function by plotting the dimensionless flow rate $Q /\left(\rho \phi_{b} W D F\left(\frac{D}{W}\right) \sqrt{g D}\right)$ as a function of the number of beads in the typical length $\ell / d$ for the two regimes (i) $\ell=D$ and (ii) $\ell=W$. In figures 14 (a) and (c) all the data for the rectangular silo are plotted and no clear trend can be observed. However, when considering each regime separately, as done in figures 14 (b) and $(\mathrm{d})$, i.e. by selecting the experiments corresponding to the first regime $\ell=D$ (and the second regime $\ell=W$ ), taking $D<1.8 W$ (respectively $D>2.2 W$ ), and considering a large number of beads in the second direction of the orifice $W>10 d$ (respectively $D>20 d$ ), the remaining data superimpose and can be well adjusted by an exponential 
$(a)$

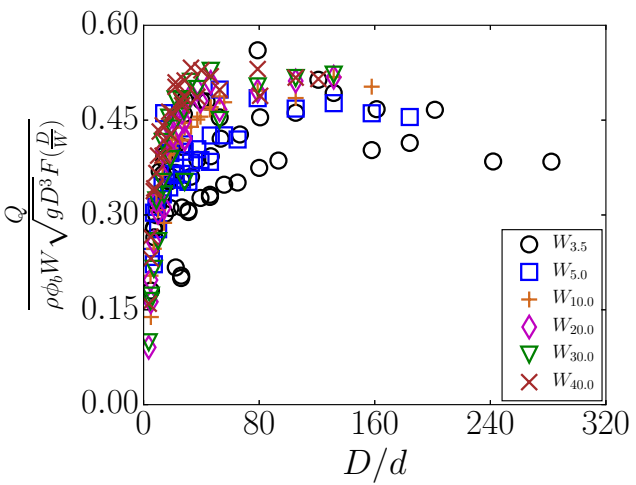

(c)

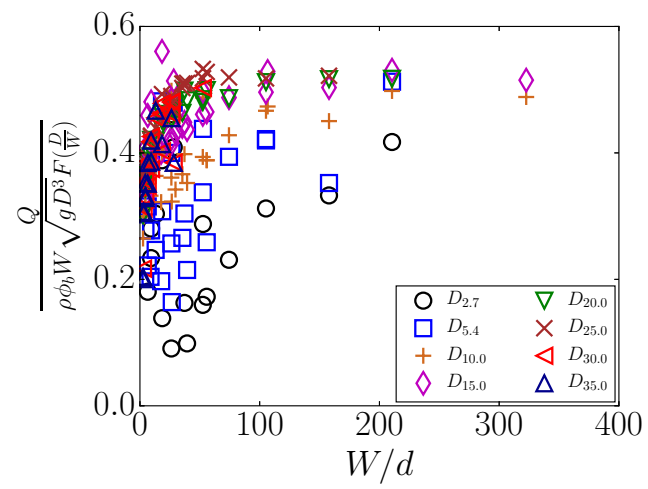

(b)

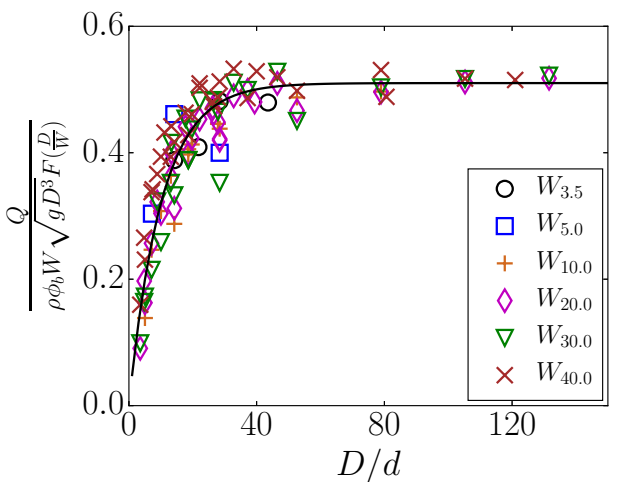

(d)

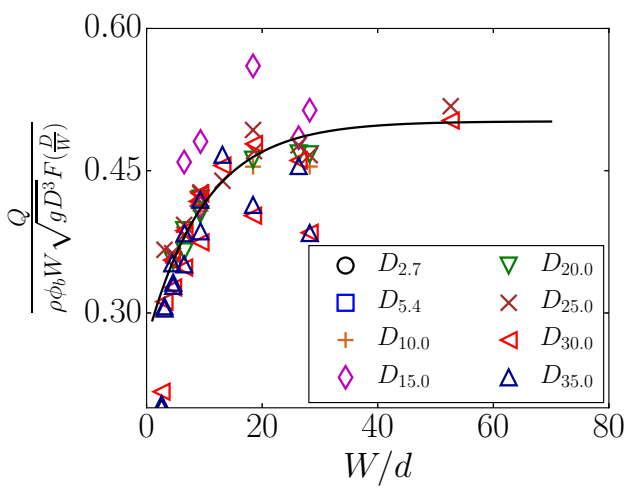

Figure 14: Experimental results obtained with rectangular silos: dimensionless flow rate $Q /\left(\rho \phi_{b} W D F\left(\frac{D}{W}\right) \sqrt{g D}\right)$ versus $(\mathrm{a}, \mathrm{b}) D / d$ and $(\mathrm{c}, \mathrm{d}) W / d$. Selected experimental runs : (a,c) all the runs, (b) $D<1.8 W$ and $W>10 d$, (d) $D>2.2 W$ and $D>20 d$. The full lines represent the geometrical functions $G_{D}(D / d)=\left[1-\alpha_{D} e^{-\beta_{D} \frac{D}{d}}\right]$ and $G_{W}(W / d)=$ $\left[1-\alpha_{W} e^{-\beta_{W} \frac{W}{d}}\right]$ with $\alpha_{D}=1, \beta_{D}=0.1, \alpha_{W}=0.46$ and $\beta_{W}=0.1$ respectively.

saturation with the fitting parameters $\alpha_{D}=1, \beta_{D}=0.1, \alpha_{W}=0.46$ and $\beta_{W}=0.1$ (see the black lines in the figures). These parameters are of the same order of magnitude as that of the literature for both geometrical functions. It is interesting to note that the same $\beta$ is recovered in the two directions. The same procedure has been followed for the cylindrical silo. Again, we find that both geometrical functions can be well adjusted by an exponential saturation, with the fitting parameters $\alpha_{D}=0.59, \beta_{D}=0.08, \alpha_{W}=0.61$, and $\beta_{W}=0.08$, in agreement with the literature.

As shown in figure $13(\mathrm{c}, \mathrm{d})$ it seems that the geometrical functions built on the two dimensions of the aperture correctly describe the dependency of the flow rate on the particle diameter. To further test some of the assumptions, we performed a $2 \mathrm{D}$ discrete simulation of the discharge flow of a silo from a lateral orifice that allows to study the effective velocity and volume fraction profiles.

\section{A.2. Discrete simulations in $2 D$}

Discrete particle simulations are described in section 2.2. In this two-dimensional configuration, following the same methodology as for the bottom orifice done 
(a)

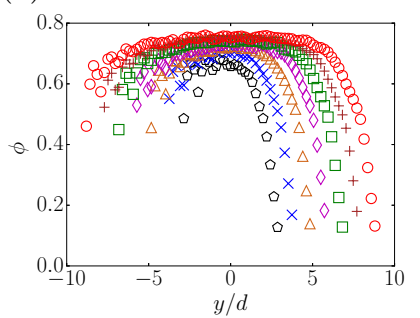

(d)

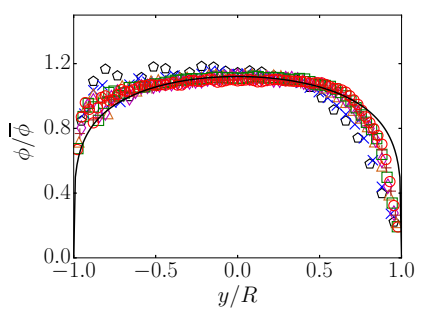

(b)

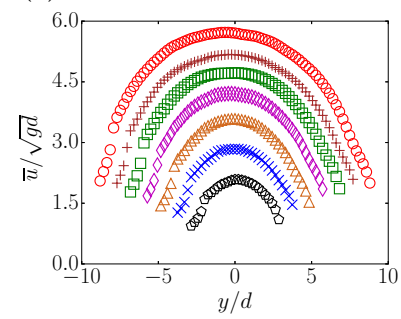

(e)

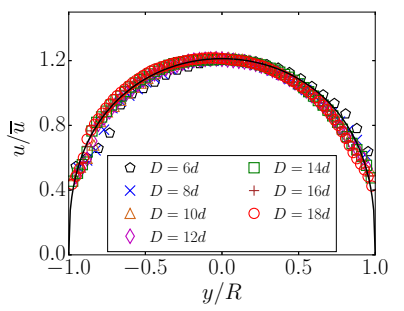

$(c)$

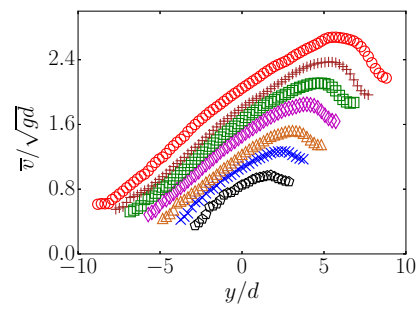

(f)

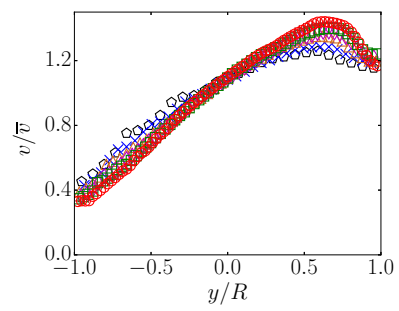

Figure 15: (Color online)(a-f) Flow of particles of diameter of $d=2 \mathrm{~mm}$ for different outlet diameters. Vertical profiles of (a) the volume fraction, $\phi,(b)$ the horizontal velocity, $u$, and (b) the vertical velocity, $v$, versus the vertical position, $y$. Vertical profiles of (d) the volume fraction normalised by the mean volume fraction, $\bar{\phi}$, (e) the horizontal velocity made dimensionless by the mean horizontal velocity, $\bar{u}$, and (f) the vertical velocity made dimensionless by the mean vertical velocity, $\bar{v}$, versus the position normalised by the outlet radius $(R=D / 2)$. The full lines represent in (d) Eq. A 3 with $\nu_{\phi}=0.21$ and in (e) Eq. A 4 with $\nu_{v}=0.38$ respectively.

experimentally by Janda et al. (2012), and with a 2D discrete simulations by Zhou et al. (2015), we are able to test most of the hypotheses $(i)$ to $(i v)$ made in the previous section, for the Hagen-Beverloo regime.

(i) We have first assumed that at the lateral outlet, the horizontal velocity profile together with the density profile are self-similar when varying the length of the hole $R=D / 2$. Figure 15 (a-c) show the vertical profile of the volume fraction, $\phi$, of the horizontal velocity, $u$, and of the vertical velocity, $v$, for various aperture lengths, $D$, for a given particle diameter $d=2 \mathrm{~mm}$. The vertical axis, $y$, is oriented upward and its origin is located at the middle of the outlet. Similar to the case of an aperture placed at the bottom of the silo, the volume fraction profile is found to be self-similar when normalised by the mean volume fraction, $\bar{\phi}$ as shown in figure $15(\mathrm{~d})$. The self-similar profile is slightly dissymmetrical, the top of the profile exhibiting a slightly higher dilatancy at the edge than at the bottom. Nevertheless, it can be well fitted by

$$
\phi(y)=\bar{\phi} \gamma\left(\nu_{\phi}\right)\left[1-\left(\frac{y}{R}\right)^{2}\right]^{\nu_{\phi}},
$$

where $\gamma(\nu)=(2 / \sqrt{\pi}) \Gamma(\nu+3 / 2) / \Gamma(\nu+1)$. The fitting parameter, $\nu_{\phi}=0.21 \pm 0.01$, obtained using the least squares method, is similar to that obtained for a bottom aperture $\nu_{\phi}=0.19 \pm 0.01$ by Zhou et al. (2015). In the same way, once normalised by the mean horizontal velocity, $\bar{u}$, the horizontal velocity profile is found to be self-similar and well 
(a)

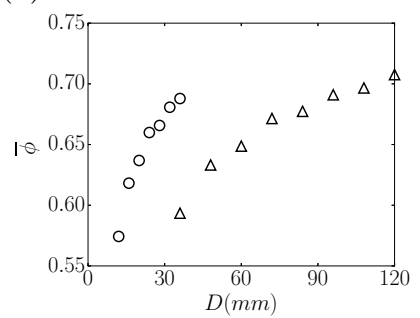

$(d)$

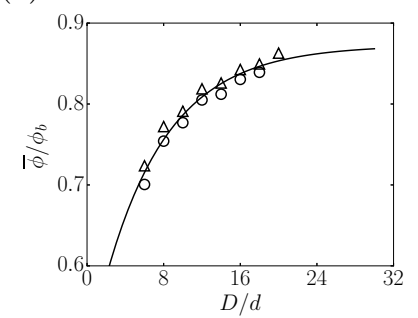

(b)

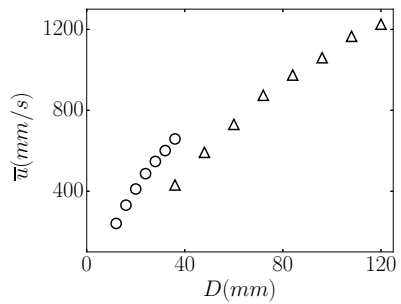

(e)

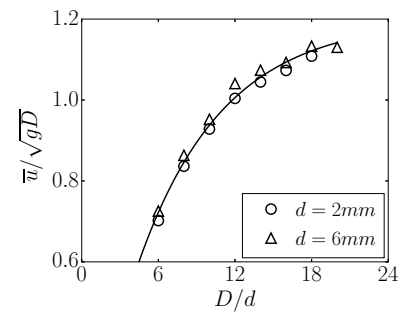

(c)

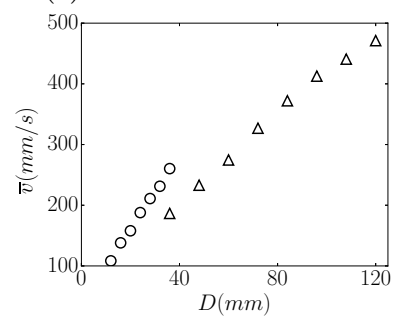

$(f)$

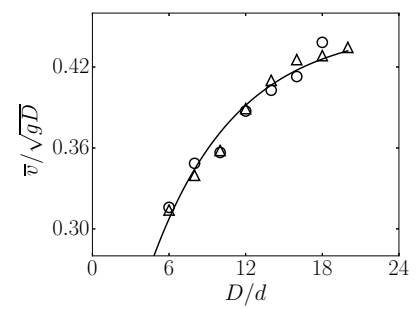

Figure 16: (a) Mean volume fraction at the outlet, $\bar{\phi}$, (b) mean horizontal velocity, $\bar{u}$, and (c) mean vertical velocity, $\bar{v}$, versus the diameter apertures $D$, for two particle diameters. (d) Mean volume fraction at the outlet, $\bar{\phi}$, normalised by the bulk volume fraction, $\phi_{b}$, (e) mean horizontal velocity, $\bar{u}$, made dimensionless by $\sqrt{g d}$ and (f) mean vertical velocity, $\bar{v}$, made dimensionless by $\sqrt{g d}$, versus the number of beads in the apertures $D / d$, for two particle diameters. The full line represent in (d) Eq. A 5 with $\xi_{\phi}=0.87, \alpha_{\phi}=0.44$, and $\beta=0.15$, in (e) Eq. A 6 with $\xi_{u}=1.2, \alpha_{u}=0.98$ and $\beta=0.15$ and in (f) Eq. A 7 with $\xi_{v}=0.45, \alpha_{v}=0.78$ and $\beta=0.15$, respectively.

adjusted by

$$
u(y)=\bar{u} \gamma\left(\nu_{v}\right)\left[1-\left(\frac{y}{R}\right)^{2}\right]^{\nu_{v}},
$$

with the fitting parameter, $\nu_{v}=0.38 \pm 0.01$ obtained using the least squares method. This parameter is identical to that obtained for the vertical velocity by Zhou et al. (2015) in the silo with a bottom aperture. Finally, the horizontal velocity profile is also found to be self-similar. The self-similar profile is clearly non-symmetric, exhibiting mainly a linear profile on the main part of outlet with a maximum close to the top where the velocity decreases toward the edge. The first hypothesis is hence fullfilled and the flow rate is given by $Q=c \rho D \bar{\phi} \bar{u}$, where $c$ is a constant.

To test the hypotheses $(i i)$ - $(i v)$, we plotted the mean volume fraction, $\bar{\phi}$ and the mean horizontal and vertical velocities, $\bar{u}$ and $\bar{v}$, as a function of the aperture length, $D$, for two particles diameters, $d=2 \mathrm{~mm}(\circ)$ and $d=6 \mathrm{~mm}(\triangle)$, as seen in figure 16 (a-c). Clearly, all the variables depend on the particle diameters. In figure 16 (d-f), the same variables are plotted, normalised by the assumed asymptotic behaviour, as a function of the number of beads in the aperture. The data collapse on single curves, as observed for the silo with a bottom aperture, which are well adjusted by an exponential saturation as 
(a)

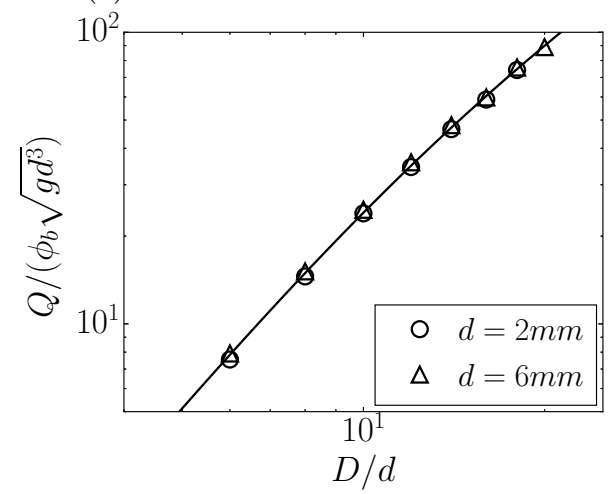

(b)

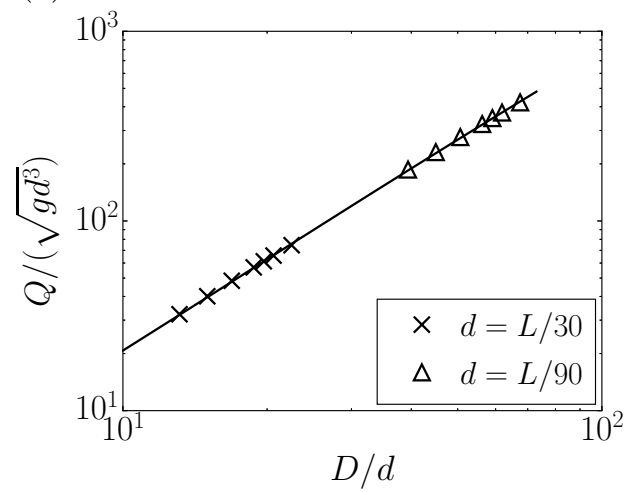

Figure 17: Flow rate made dimensionless by $\sqrt{g d^{3}}$ versus the number of beads in the aperture $D / d$ for the $2 \mathrm{D}$ case, (a) discrete simulation, (b) continuum simulation. The full lines represent Eq. A 8 with (a) $C_{l}^{\prime}=1.08$ and (b) $C_{l}^{\prime}=0.78$.

expected,

$$
\begin{aligned}
& \bar{\phi}=\xi_{\phi} \phi_{b}\left[1-\alpha_{\phi} e^{-\beta \frac{D}{d}}\right]=\xi_{\phi} \phi_{b} G_{\phi}\left(\frac{D}{d}\right), \\
& \bar{u}=\xi_{u} \sqrt{g D}\left[1-\alpha_{u} e^{-\beta \frac{D}{d}}\right]=\xi_{u} \sqrt{g D} G_{u}\left(\frac{D}{d}\right), \\
& \bar{v}=\xi_{v} \sqrt{g D}\left[1-\alpha_{v} e^{-\beta \frac{D}{d}}\right]=\xi_{v} \sqrt{g D} G_{v}\left(\frac{D}{d}\right),
\end{aligned}
$$

with the fitting parameters $\beta=0.15, \xi_{\phi}=0.87, \alpha_{\phi}=0.44, \xi_{u}=1.2, \alpha_{u}=0.98, \xi_{v}=$ $0.45, \alpha_{v}=0.78$, obtained using the least-squares method. Once again, these parameters closely match those obtained by Zhou et al. (2015) for the bottom configuration. The same $\beta$, fitted on the volume fraction, is found to correctly adjust the mean velocity variations. This suggests that the same phenomenon is involved in the variation at the outlet of the volume fraction and of the velocities with respect to the particle size. These equations also suggest that in the Hagen-Beverloo regime, the angle of inclination of the streamline at the outlet, defined as $\tan (\theta)=\bar{u} / \bar{v}$ has low dependency on the outlet size $D$ through a geometrical function $G_{u} / G_{v}$. Finally the flow rate is given by

$$
Q=C_{l}^{\prime} \rho \phi_{b} \sqrt{g D} G_{\phi}\left(\frac{D}{d}\right) G_{u}\left(\frac{D}{d}\right) \approx C_{l}^{\prime} \rho \phi_{b} \sqrt{g D} G\left(\frac{D}{d}\right),
$$

where $C_{l}^{\prime}=\xi_{\phi} \xi_{v} \gamma\left(\nu_{v}\right) \gamma\left(\nu_{\phi}\right) \int_{0}^{1}\left(1-t^{2}\right) d t=1.08$, and $G=\left[1-\alpha e^{-\beta \frac{D}{d}}\right]$ with $\alpha=\alpha_{\phi}+\alpha_{u}$. In the range of number of beads in the aperture $(D / d>6)$, the approximation of $G \approx$ $G_{u} G_{\phi}$ leads to less than $5 \%$ of error. This equation adjusts the data well with the fitting parameter $C_{l}^{\prime}=1.08$, as seen in figure 17 , and is similar to equation A 2 considering $W=\infty$.

Using 2D discrete simulations we have shown that the dependence of the flow rate on the particle size can be modeled using a geometrical function which depends only on the number of beads in the aperture. This geometrical function is seen to influence both the volume fraction at the outlet, bigger particles leading to a dilation, and the velocity, bigger particles leading to slower flow. It would be interesting in future work to conduct $3 \mathrm{D}$ discrete simulations to see if we recover the same geometrical function for the number 
(a)

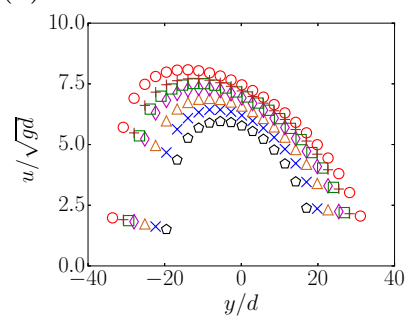

(d)

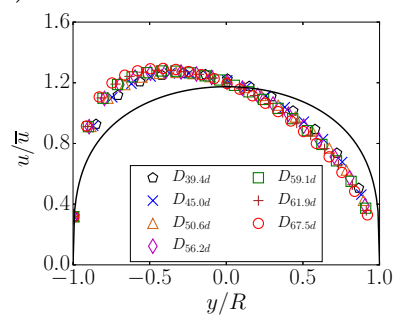

(b)

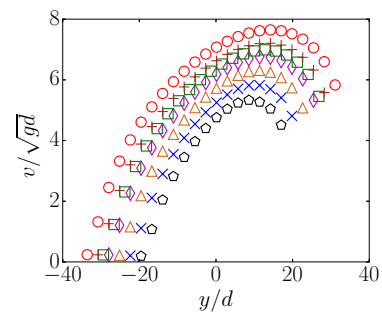

(e)

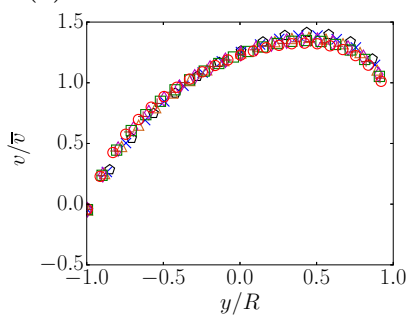

(c)
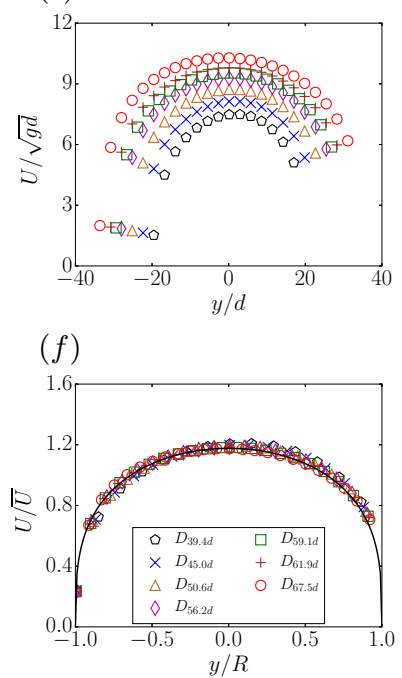

Figure 18: 2D continuum simulation (a,d) horizontal velocity (b,e) vertical velocity and $(\mathrm{c}, \mathrm{f})$ norm of the velocity, $(\mathrm{a}, \mathrm{b}, \mathrm{c})$ normalised by $\sqrt{g d}$ versus $y / d$ and $(\mathrm{d}, \mathrm{e}, \mathrm{f})$ normalised by the mean value versus $y / R$, the full lines in $(\mathrm{d}, \mathrm{f})$ represent Eq. A 4 with $\nu_{v}=0.31$.

of beads in the silo width, in the regime dominated by the lateral friction, as suggested by the experimental results.

\section{A.3. Continuum simulation}

We have seen in section 3 that the continuum simulation described correctly most of the observations on the discharge of a silo with a lateral aperture, for various $D$ and various $W$ but for a given particle diameter. However the $\mu(I)$ rheology contains the information on the particle diameter in the definition of the inertial number $I=d \sqrt{2} D_{2} /(\sqrt{p / \rho})$. In this section we wonder if the continuum simulation is able to recover partially the dependency on the particle size of the flow rate. Indeed, even if this simulation considers a constant volume fraction, the $2 \mathrm{D}$ discrete simulations have shown that the velocity at the outlet may follow the same geometrical relation than the volume fraction.

Figure 18 presents the vertical profile at the outlet of the horizontal velocity, the vertical velocity and the norm of the velocity, for a given particle diameter in the $2 \mathrm{D}$ case. Interestingly, when normalised by the mean value, these profiles are again found to be self-similar. However, contrary to the discrete simulations, the horizontal velocity profile exhibits a strong asymmetry between the top and the bottom of the outlet. As a consequence, the ajustement by the equation A 4 with the fitting parameter $\nu_{u}=$ $0.31 \pm 0.01$ obtained using least squares method is not satisfactory. However the vertical velocity profile ressembles the discrete simulation result. Interestingly, the profile of the norm of the velocity is found to remain symmetrical and is well adjusted by equation Eq. A 4 with $\nu_{v}=0.31$.

Figures 19(a,b,c) show the mean value of the horizontal and vertical velocities and of the norm of the velocity, normalised by $\sqrt{g L}$ versus the dimensionless outlet diameter $D / d$ for two particles diameters $d=L / 30$ and $d=L / 90$. Clearly these mean velocities depend on the outlet size $D$, but also on the particle diameter $d$. Following the discrete simulation study we have plotted in figure $19(\mathrm{~d}, \mathrm{e}, \mathrm{f})$ these velocities made dimensionless with $\sqrt{g D}$ 
(a)

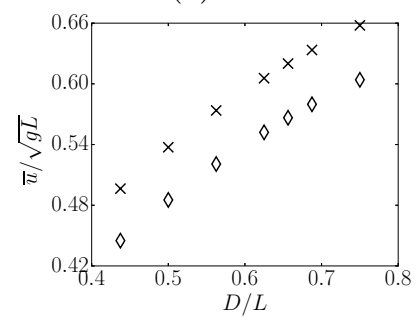

$(d)$

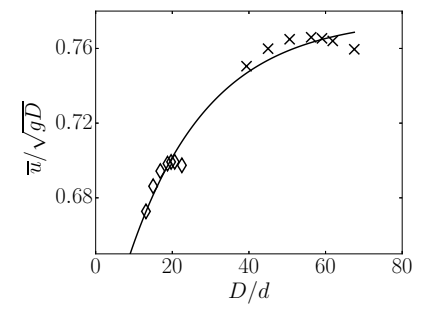

(b)

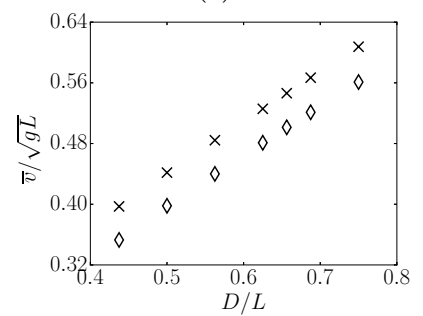

$(e)$

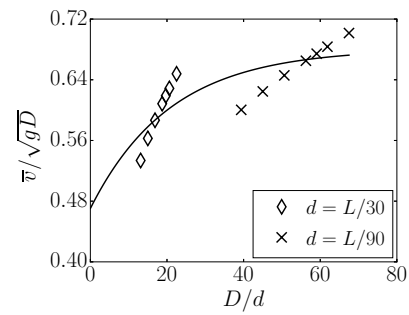

$(c)$

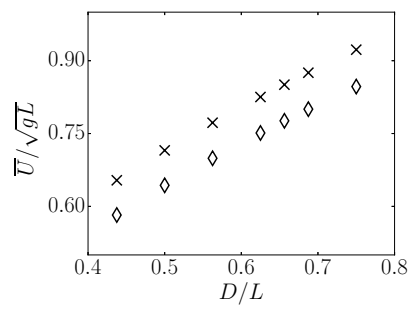

$(f)$

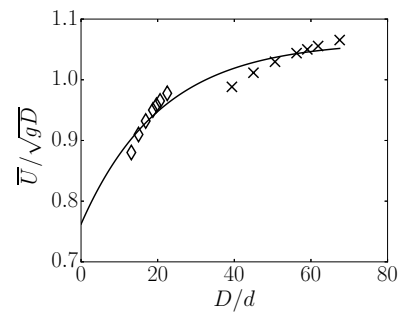

Figure 19: 2D continuum simulation (a,d) horizontal velocity, (b,e) vertical velocity, and $(\mathrm{c}, \mathrm{f})$ norm of the velocity normalised in $(\mathrm{a}, \mathrm{b}, \mathrm{c})$ by $\sqrt{g L}$ versus $D / L$ and normalised in (d,e,f) by $\sqrt{g D}$ versus $D / d$ for two particles diameters. The full lines in (d,e,f) represent equation A 6 with the fitting parameters $\beta=0.05, \xi_{u}=0.78, \alpha_{u}=0.25, \xi_{v}=0.68$, $\alpha_{v}=0.31, \xi_{U}=1.06$ and $\alpha_{U}=0.28$ obtained using the least squares method.

versus $D / d$. The data corresponding to the mean horizontal velocity (respectively to the mean norm) are reasonably well adjusted by equation A 6 with the fitting parameters $\beta=0.05, \xi_{u}=0.78$ and $\alpha_{u}=0.25$ (respectively $\xi_{U}=1.06$ and $\alpha_{U}=0.28$ ). The value of $\beta$ is sensibly lower than the value obtained experimentally or in the discrete simulation. However the same tendency than observed in the discrete simulation is recovered. This is not the case for the mean vertical velocity for which the data do not follow the asymptotic behaviour in $\sqrt{g D}$. However this velocity is not involved in the flow rate and the equation A 8 using the fitting parameters predicts well the flow rate, see the full line in figure $17 \mathrm{~b}$.

Finally we have done the same analysis in the pseudo-3D continuum simulation, in the regime controlled by the sidewall friction. Figure 20a presents the profile of the horizontal velocity at the outlet for various $D$. In this regime, the profiles present the same asymmetric shape than in the Hagen-Beverloo profile, nevertheless the velocity does not seem to depend on $D$ anymore. Figure $20 \mathrm{~b}$ shows the mean horizontal velocity, normalised by $\sqrt{g W}$ versus $W / d$ for data corresponding to the second regime, $D / W>3$. Clearly we do not observe the geometrical function as suggested by the experimental results. This is not surprising as the flow is not solved throughout the silo width, thus the $I$ number is not expected to play any role in this direction.

To conclude this section, we found that a dependency on the particle diameter is observed in the $2 \mathrm{D}$ continuum simulation, that the velocity profiles are self-similar when varying the outlet length, and that the mean horizontal velocity tends asymptotically towards $\sqrt{g D}$, contrary to the mean vertical velocity. This behaviour is well described by a geometrical function given by equation A 6 . However the profiles present a larger asymmetry than the one predicted by the $2 \mathrm{D}$ discrete simulations. This can be due to the fact that the volume fraction has been assumed to be uniform, whereas the complete $\mu(I)$-rheology, that includes a $\phi(I)$ relation, would predict a dilation of a granula media 
(a)

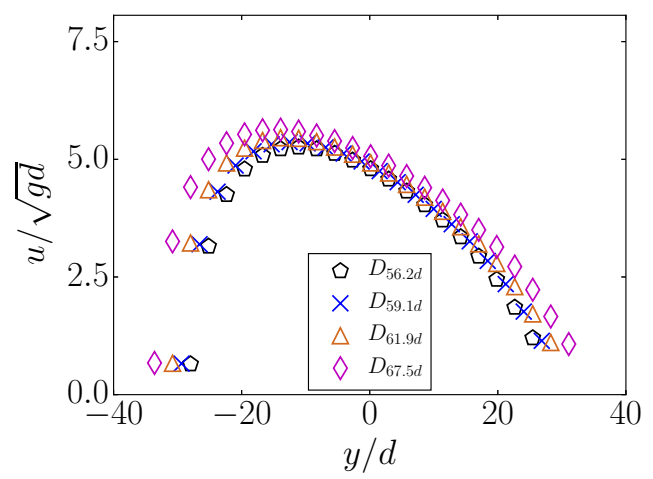

(b)

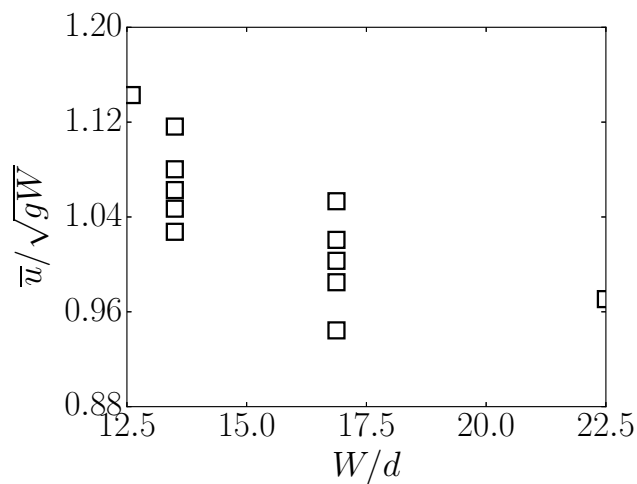

Figure 20: Pseudo-3D continuum simulation. (a) Horizontal velocity normalized by $\sqrt{g d}$ versus $y / d$ at the outlet for different $D$ and $W=13.5 d$ (b) mean horizontal velocity normalised by $\sqrt{g W}$ for $D / W>3$.

when it is sheared. The dependency on the particle diameter for the regime controlled by the sidewalls is not correctly described by the pseudo-3D continuum simulation which does not solve the flow in the silo width direction. 